\title{
Convex Risk Measures: Basic Facts, Law-invariance and beyond, Asymptotics for Large Portfolios
}

\author{
Hans Föllmer $\quad$ Thomas Knispel ${ }^{\mathrm{b}}$
}

\begin{abstract}
This paper provides an introduction to the theory of capital requirements defined by convex risk measures. The emphasis is on robust representations, law-invariant convex risk measures and their robustification in the face of model uncertainty, asymptotics for large portfolios, and on the connections of convex risk measures to actuarial premium principles and robust preferences.
\end{abstract}

Key words: Monetary risk measure, convex and coherent risk measure, capital requirement, acceptance set, risk functional, deviation measure, robust representation, law-invariant risk measure, Value at Risk, Stressed Value at Risk, Average Value at Risk, shortfall risk measure, divergence risk measure, Haezendonck risk measure, entropic risk measure, model uncertainty, robustified risk measure, asymptotics for large portfolios, robust large deviations, actuarial premium principles, Esscher principle, Orlicz premium principle, Wang's premium principle, robust preferences

Mathematics Subject Classification (2010): 46E30, 60F10, 62P05, 91B08, 91B16, 91B30

\section{Introduction}

The quantification of financial risk takes many forms, depending on the context and the purpose. Here we focus on monetary measures of risk which take the form of a capital requirement: The risk $\rho(X)$ of a given financial position $X$ is specified as the minimal capital that should be added to the position in order to make that position acceptable.

A financial position will be described by its uncertain net monetary outcome at the end of a given trading period, and so it will be modeled as a real-valued measurable function $X$ on a measurable space $(\Omega, \mathcal{F})$ of possible scenarios. We fix a linear space $\mathcal{X}$ of such positions and a subset $\mathcal{A} \subset \mathcal{X}$ of positions which are defined to be acceptable. Our focus will be on the downside risk, and so we require that $Y \in \mathcal{A}$ whenever $Y \geq X$ for some $X \in \mathcal{A}$. The functional $\rho$ on $\mathcal{X}$ defined by

$$
\rho(X):=\inf \{m \in \mathbb{R} \mid X+m \in \mathcal{A}\}
$$

is then called a monetary risk measure.

The standard example of a monetary risk measure is Value at Risk at some level $\lambda$. Here it is assumed that there is a probability measure $P$ on the underlying space of scenarios, and that we have sufficient access to it through past observations. A position $X$ is now defined to be

\footnotetext{
Acknowledgement: This paper was written in part while the first author was visiting ETH Zurich, funded by the Forschungsinstitut für Mathematik (FIM); this support is gratefully acknowledged.

${ }^{a}$ Humboldt-Universität zu Berlin, Institut für Mathematik, Unter den Linden 6, 10099 Berlin, Germany, email: foellmer@math.hu-berlin.de

${ }^{\mathrm{b}}$ Leibniz Universität Hannover, Institut für Mathematische Stochastik, Welfengarten 1, 30167 Hannover, Germany, email: knispel@stochastik.uni-hannover.de
} 
acceptable if the probability $P[X<0]$ of a shortfall does not exceed the level $\lambda$. The resulting capital requirement $\operatorname{VaR}_{\lambda}(X)$ is then given, up to a minus sign, by a $\lambda$-quantile of the distribution of $X$ under $P$. Although widely used in practice, Value at Risk has a number of deficiencies. In particular, it may penalize diversification, and it does not pay attention to the size of the shortfall. Some of these deficiencies were recognized early on, and they motivated the axiomatic approach to a general theory of monetary risk measures, which was initiated by Artzner, Delbaen, Eber and Heath in the late nineties, and which is the topic of this survey. Other drawbacks became apparent during the recent financial crisis; cf. the careful analysis of Value at Risk and its procyclical effects in The Turner Review - A regulatory response to the global banking crisis [1. In particular, the Turner Review points to an excessive reliance on a single probabilistic model $P$. More generally, it raises the issue of model uncertainty or model ambiguity, often called Knightian uncertainty. As discussed below, the theory of risk measures can in fact be seen as a case study which explores how to deal with this Knightian uncertainty in mathematical terms.

In order to capture the idea that diversification is desirable and should not be penalized by the capital requirements, we require that the acceptance set $\mathcal{A}$ should be convex. In this case the monetary risk measure $\rho$ is called a convex risk measure, because convexity of $\mathcal{A}$ is equivalent to convexity of $\rho$, viewed as a functional on the linear space $\mathcal{X}$ of financial positions. If a position remains acceptable even if it is scaled up by any $\lambda>0$, then $\mathcal{A}$ is a convex cone, and the risk measure $\rho$ is called a coherent risk measure. In the context of mathematical finance, coherent risk measures first appear in the seminal paper by Artzner, Delbaen, Eber \& Heath [7. The subsequent extension from coherent to convex risk measures was introduced independently in Frittelli \& Rosazza Gianin [4], Heath [56], and Föllmer \& Schied [41].

Convex duality shows that a convex risk measure typically takes the following form

$$
\rho(X)=\sup _{Q \in \mathcal{Q}_{\rho}}\left\{E_{Q}[-X]-\alpha(Q)\right\},
$$

where $\mathcal{Q}_{\rho}$ is some class of plausible probability measures on the underlying set of possible scenarios, and $\alpha$ is some penalty function on $\mathcal{Q}_{\rho}$. The capital requirement is thus determined as follows: The expected loss of a position is calculated for the probability measures in $\mathcal{Q}_{\rho}$. But these models are taken seriously to a different degree as prescribed by the penalty function, and so one takes the worst penalized expected loss over the class $\mathcal{Q}_{\rho}$. In this way, model uncertainty is taken into account explicitly. In the special coherent case the penalty function will vanish on $\mathcal{Q}_{\rho}$, and so the representation (1) reduces to

$$
\rho(X)=\sup _{Q \in \mathcal{Q}_{\rho}} E_{Q}[-X],
$$

i. e., to the worst case expected loss over the class $\mathcal{Q}_{\rho}$.

In the context of mathematical finance, the history of coherent and convex risk measures begins with the seminal paper [7, as mentioned above. In a wider mathematical perspective, however, there is a considerable pre-history in areas such as the theory of Choquet integrals, robust statistics, actuarial premium principles, and the microeconomic theory of preferences:

- In robust statistics, the axiomatic definition of coherent risk measures appears under the name of "upper expectations" in Huber [58, Chapter 10.2, together with the representation 22), although this is proved only in the special case of a finite set of scenarios; cf. also Huber \& Strassen [59.

- In actuarial mathematics, convex risk measures clearly appear, up to a change of sign, as convex principles of premium calculation in Deprez \& Gerber [28], but without their robust representation (1). Moreover, there is a considerable literature on comonotonic premium principles where the implicit risk measure is coherent and can be described as a Choquet integral with respect to a concave distortion of some underlying probability measure $P$; cf., e. g., Panjer, Young \& Wang [71] and Dhaene et al. [30]. 
- In the context of mathematical economics, preferences on $\mathcal{X}$ are usually represented by some utility functional $U$ on $\mathcal{X}$. Under the axioms of rationality as formulated by von Neumann \& Morgenstern [80] and Savage [77], $U$ takes the form of an expected utility, i. e.,

$$
U(X)=E_{P}[u(X)]
$$

for some increasing continuous function $u$ and some probability measure $P$ on $(\Omega, \mathcal{F})$. As shown by Gilboa \& Schmeidler [50] in the late eighties, a natural relaxation of the axioms of rationality implies that the linear risk measure $\rho=-E_{P}$ in $(3)$ should be replaced by a general coherent risk measure:

$$
U(X)=-\rho(u(X))=\inf _{Q \in \mathcal{Q}_{\rho}} E_{Q}[u(X)]
$$

More recently, Maccheroni, Marinacci \& Rustichini 68] have relaxed the rationality axioms even further. In their axiomatic setting, $\rho$ is now a convex risk measure, and so the numerical representation of preferences takes the form

$$
U(X)=-\rho(u(X))=\inf _{Q \in \mathcal{Q}_{\rho}}\left\{E_{Q}[u(X)]+\alpha(Q)\right\}
$$

As illustrated by the preceding case studies, convex risk measures arise naturally in a wide variety of applications, and their interest is not limited to the area of financial risk management. In this partial survey, our aim is twofold. On the one hand, we intend to provide a concise introduction to basic facts in the mathematical theory of convex risk measures, and to illustrate their relevance by various examples and applications. On the other hand, we want to describe some recent developments related to the robustification of law-invariant convex risk measures and to their asymptotic behavior for large portfolios.

The paper is organized as follows. In Section 2 we introduce convex and coherent risk measures and discuss their connection to general quasi-convex risk functionals. Section 3 focuses on the representation of convex risk measures as worst-case penalized expected loss in the sense of (1), in particular on the Banach spaces of bounded measurable or bounded continuous functions, on the Banach spaces $L^{p}(\Omega, \mathcal{F}, P), 1 \leq p \leq \infty$, and on Orlicz hearts. In Section 4 we describe a variety of examples, including Value at Risk, Average Value at Risk, shortfall risk measures, divergence risk measures, Haezendonck risk measures, and different classes of entropic risk measures. All these examples are law-invariant in the sense that the capital requirement $\rho(X)$ only depends on the law of $X$ under some given probability measure $P$. In Section 5 we describe the general structure of such law-invariant risk measures in terms of mixtures of Average Value at Risk. Moreover, we discuss the interplay between law-invariance and stochastic dominance, the characterization of comonotonic risk measures as Choquet integrals, the interpretation of risk measures as functionals on lotteries, and the connection between risk measures and generalized deviations in the sense of Rockafellar, Uryasev \& Zabarankin [74]. In Section 6] we return to the issue of Knightian uncertainty, or model ambiguity. Here we introduce robustified versions of law-invariant risk measures, where the single probability measure $P$ is replaced by a whole class $\mathcal{P}$ of probabilistic models. In particular, we consider robustified versions of different types of entropic risk measures and describe the behavior of the corresponding capital requirements for large portfolios. A general discussion of convex capital requirements for large portfolios is given in Section 7, first in the law-invariant case and then in the face of model ambiguity. In Section 8 we explain the close connection between convex risk measures and actuarial premium principles, and the final Section 9 is devoted to robust preferences and their relation to convex risk measures.

Let us emphasize that this paper is only a partial survey: Many important topics are not covered here. In particular we limit the discussion to a static setting where risk measures are applied to real-valued functions on some set of scenarios $\Omega$. For an introduction to conditional risk measures and their dynamic behavior along a given filtration we refer to Föllmer \& Schied [42], 
Chapter 11, and the references therein; cf. also Acciaio \& Penner [4. Risk measures for stochastic processes can be discussed within the static setting, since adapted processes with parameter set $T$ can be identified with real-valued functions that are measurable with respect to the optional $\sigma$-field on $\Omega \times T$. But this requires a careful analysis of the probability measures on the optional $\sigma$-field which appear in the robust representation and describe the interplay between model uncertainty and uncertainty about the time-value of money; cf., for example, Acciaio, Föllmer \& Penner [3] and Föllmer \& Penner 40 and the references therein. Another important topic which is not covered here is the study of stochastic optimization problems formulated in terms of risk measures; here we refer to Pflug \& Römisch 73 and also to Föllmer, Schied \& Weber [43].

\section{Risk measures and their acceptance sets}

A financial position will be described by its monetary outcome, and this outcome is typically uncertain. More precisely, we fix a measurable space $(\Omega, \mathcal{F})$ of possible scenarios and define a financial position as a measurable function $X: \Omega \rightarrow \mathbb{R}$, where $X(\omega)$ is the discounted net worth of the position at the end of a given trading period if the scenario $\omega \in \Omega$ is realized. Our aim is to quantify the monetary risk of the position $X$ as the additional capital $\rho(X)$ that is required to make the position acceptable, for example from the point of view of a supervising agency. This leads us to the notion of a monetary risk measure, defined as a functional $\rho$ on a given class $\mathcal{X}$ of financial positions.

From now on $\mathcal{X}$ will denote a linear space of measurable functions on $(\Omega, \mathcal{F})$ that contains the constants.

Definition 2.1. A functional $\rho: \mathcal{X} \rightarrow \mathbb{R} \cup\{\infty\}$ with $\rho(0) \in \mathbb{R}$ is called a monetary risk measure if it is

i) monotone, i. e., $\rho(X) \leq \rho(Y)$ if $X \geq Y$,

and

ii) cash-invariant, i. e., $\rho(X+m)=\rho(X)-m$ for $X \in \mathcal{X}$ and $m \in \mathbb{R}$.

If $\rho$ is a monetary risk measure, then the set

$$
\mathcal{A}_{\rho}:=\{X \in \mathcal{X} \mid \rho(X) \leq 0\}
$$

is called the acceptance set of $\rho$, and $\rho$ can be recovered from the acceptance set via

$$
\rho(X)=\inf \left\{m \in \mathbb{R} \mid X+m \in \mathcal{A}_{\rho}\right\} .
$$

Thus, a monetary risk measure can be viewed as a capital requirement: $\rho(X)$ is the minimal capital that has to be added to the position $X$ to make it acceptable. The acceptance set $\mathcal{A}_{\rho}$ is nonempty and has the following two properties:

- $\inf \left\{m \in \mathbb{R} \mid m \in \mathcal{A}_{\rho}\right\}>-\infty$,

- $X \in \mathcal{A}_{\rho}, Y \in \mathcal{X}, Y \geq X \quad \Longrightarrow \quad Y \in \mathcal{A}_{\rho}$.

Conversely, any nonempty subset $\mathcal{A}$ of $\mathcal{X}$ that satisfies these two conditions defines via (5) a monetary risk measure $\rho$ with acceptance set $\mathcal{A}_{\rho}=\mathcal{A}$.

In order to capture the idea that diversification should not increase the risk, it is natural to require quasi-convexity of $\rho$, i. e.,

$$
\rho(\lambda X+(1-\lambda) Y) \leq \max \{\rho(X), \rho(Y)\}
$$


for $X, Y \in \mathcal{X}$ and $\lambda \in(0,1)$. In that case, $\mathcal{A}_{\rho}$ is convex, and this implies that $\rho$ is in fact a convex functional on $\mathcal{X}$, i. e., $\rho$ satisfies not only (6) but the stronger inequality

$$
\rho(\lambda X+(1-\lambda) Y) \leq \lambda \rho(X)+(1-\lambda) \rho(Y) ;
$$

cf., e. g., [42], Proposition 4.6.

Definition 2.2. A monetary risk measure is called a convex risk measure if it satisfies the condition (6) of quasi-convexity and is hence convex. A convex risk measure is called coherent if it is also positively homogeneous, i.e.,

$$
\rho(\lambda X)=\lambda \rho(X)
$$

for $X \in \mathcal{X}$ and $\lambda \geq 0$.

Any coherent risk measure $\rho$ is normalized in the sense that $\rho(0)=0$, and its acceptance set $\mathcal{A}_{\rho}$ is a convex cone. Note also that a coherent risk measure $\rho$ is subadditive, i. e.,

$$
\rho(X+Y) \leq \rho(X)+\rho(Y)
$$

for $X, Y \in \mathcal{X}$. More generally: If $\rho$ is a normalized monetary risk measure then any two of the three properties of convexity, positive homogeneity, and subadditivity imply the remaining third; cf., e. g., [42, Exercise 4.1.3.

Remark 2.1. In the preceding definitions it is usually assumed that all financial positions are already discounted by the risk-free interest rate $r$. If the risk of the undiscounted position $\tilde{X}:=$ $(1+r) X$ is specified by $\tilde{\rho}(\tilde{X})=\rho(X)$ then the resulting functional $\tilde{\rho}$ is still monotone. But cashinvariance now takes the form

$$
\tilde{\rho}(\tilde{X}+(1+r) m)=\tilde{\rho}(\tilde{X})-m,
$$

i. e., adding $m$ units of money to the portfolio at time 0 and investing it in a risk-free asset reduces the capital requirement by $m$.

One may want to relax the restriction that the supporting capital can only be invested in a risk-free asset, and to allow investment in some larger class $\mathcal{V} \subset \mathcal{X}$ of "admissible" assets. This case can be reduced to our present setting by enlarging the initial acceptance set $\mathcal{A}$ to some larger set $\mathcal{A}_{\mathcal{V}}$; cf. Föllmer 8$\}$ Schied [41] or [42], Section 4.8, and Artzner, Delbaen 83 Koch-Medina [8]. If the initial risk measure $\rho$ is coherent but the assets in $\mathcal{V}$ are subject to convex trading constraints, then the resulting risk measure $\rho_{\mathcal{V}} \leq \rho$ will only be convex. This was one of the reasons in [41] to introduce the general notion of a convex risk measure.

In this survey we focus on monetary risk measures and on their interpretation as capital requirements. Note, however, the following connection to more general notions of the "downside risk" which are obtained by dropping the condition of cash-invariance in Definition 2.1

Definition 2.3. Let us call $\rho$ a risk functional if it is monotone as in Definition 2.1 and quasiconvex as in (6).

Without the condition of cash-invariance we can no longer conclude that $\rho$ is convex. But $\rho$ can be identified with a family of convex risk measures in the following manner. Consider any functional $\rho: \mathcal{X} \rightarrow(-\infty, \infty]$ such that $\rho \not \equiv \infty$ and $\lim _{m \downarrow-\infty} \rho(m)=\infty$. For each $r>\underline{r}:=\inf \rho$ the set

$$
\mathcal{A}_{r}:=\{X \in \mathcal{X} \mid \rho(X) \leq r\}
$$

is an acceptance set, and so it defines via (5) a monetary risk measure $\rho_{r}$ given by

$$
\rho_{r}(X)=\inf \{m \mid \rho(X+m) \leq r\} .
$$


The family $\left(\mathcal{A}_{r}\right)$ is increasing in $r>\underline{r}$ while $\left(\rho_{r}\right)$ is decreasing, and the risk functional $\rho$ can be reconstructed from $\left(\mathcal{A}_{r}\right)$ or $\left(\rho_{r}\right)$ via

$$
\rho(X)=\inf \left\{r>\underline{r} \mid X \in \mathcal{A}_{r}\right\}=\inf \left\{r>\underline{r} \mid \rho_{r}(X) \leq 0\right\} .
$$

Moreover, quasi-convexity of $\rho$ implies that each $\mathcal{A}_{r}$ is convex, and so the corresponding monetary risk measure $\rho_{r}$ is in fact convex. Thus any risk functional $\rho$ corresponds to a family of convex risk measures. We refer to Drapeau [31, Brown, De Giorgi \& Sim [14, and Drapeau \& Kupper [32] for a systematic discussion and a wide variety of case studies.

Remark 2.2. We limit our discussion to risk measures for real-valued financial positions $X$. But it is also natural to consider risk measures for portfolio vectors $X=\left(X_{1}, \ldots, X_{n}\right)$. In this case, each component can be seen as the monetary outcome of a subportfolio, and a risk measure for portfolio vectors allows to quantify the monetary risk caused by the variation of its components and by their dependence. For a systematic discussion we refer to Jouini, Meddeb 63 Touzi [60], Burgert \& Rüschendorf [17], Rüschendorf [75] and the references therein.

\section{Robust representations}

Let us denote by $\mathcal{M}_{1}$ the set of all probability measures on $(\Omega, \mathcal{F})$.

Definition 3.1. We say that a convex risk measure $\rho$ has a robust representation if

$$
\rho(X)=\sup _{Q \in \mathcal{M}_{1}}\left\{E_{Q}[-X]-\alpha(Q)\right\}=\sup _{Q \in \mathcal{Q}_{\rho}}\left\{E_{Q}[-X]-\alpha(Q)\right\}
$$

with some penalty function $\alpha: \mathcal{M}_{1} \rightarrow \mathbb{R} \cup\{\infty\}$, where

$$
\mathcal{Q}_{\rho}:=\left\{Q \in \mathcal{M}_{1} \mid \alpha(Q)<\infty\right\}
$$

is such that $E_{Q}[-X]$ is well-defined for $Q \in \mathcal{Q}_{\rho}$ and $X \in \mathcal{X}$.

Note that 99 implies

$$
\inf _{Q \in \mathcal{M}_{1}} \alpha(Q)>-\infty
$$

since $\rho(0)$ is finite. Conversely, any such penalty function defines a convex risk measure via (9).

The measures in $\mathcal{Q}_{\rho}$ can be seen as plausible probabilistic models, taken more or less seriously as specified by the penalty function $\alpha$. The capital requirement $\rho(X)$ is then computed as the worst case of the penalized expected loss $E_{Q}[-X]$, taken over all models $Q \in \mathcal{Q}_{\rho}$. Note that the general definition of a convex risk measure does not a priori involve the choice of a probabilistic model. But probabilistic models do come in through the robust representation, playing the role of stress tests.

If $\rho$ admits a robust representation (9) in terms of the penalty function $\alpha$, then the representation also holds for the penalty function $\alpha^{\text {min }}$ defined by

$$
\alpha^{\min }(Q):=\sup _{X \in \mathcal{X}}\left\{E_{Q}[-X]-\rho(X)\right\}=\sup _{X \in \mathcal{A}_{\rho}} E_{Q}[-X] .
$$

Note that $\alpha^{\min }(Q) \leq \alpha(Q)$, since $(9)$ implies $\alpha(Q) \geq E_{Q}[-X]-\rho(X)$ for all $X \in \mathcal{X}$.

In the coherent case we have $\alpha^{\min }(Q) \in\{0, \infty\}$, since the acceptance set $\mathcal{A}_{\rho}$ is a convex cone. A coherent risk measure $\rho$ with robust representation 9 thus takes the form

$$
\rho(X)=\sup _{Q \in \mathcal{Q}_{\rho}} E_{Q}[-X]
$$


with $\mathcal{Q}_{\rho}=\left\{Q \in \mathcal{M}_{1} \mid \alpha^{\min }(Q)=0\right\}$; cf. Artzner, Eber, Delbaen \& Heath [7], Delbaen [25], and [42], Corollaries 4.19 and 4.37 .

The second expression in 10 describes $\alpha^{\min }(Q)$ as the worst expected loss under $Q$ for any acceptable position. The first expression identifies $\alpha^{\min }(Q)$ as the Fenchel-Legendre transform of the convex functional $\rho$, applied to linear functionals of the form $l(X)=E_{Q}[-X]$ for $Q \in \mathcal{M}_{1}$. It is therefore plausible that the robust representation (9) should follow from the Fenchel-Moreau theorem, applied to the convex functional $\rho$ under appropriate topological assumptions on $\mathcal{X}$ and $\rho$. Indeed, the monetary properties of $\rho$ guarantee that any linear form $l$ on $\mathcal{X}$ with finite FenchelLegendre transform satisfies $-l \geq 0$ and $-l(1)=1$, and this suggests that $l$ should be of the form $l(X)=E_{Q}[-X]$ for some probability measure $Q$. We now describe some specific situations where this heuristic argument can be turned into a rigorous proof; for a systematic discussion in a very general framework we refer to Frittelli \& Rosazza Gianin [44] and Biagini \& Frittelli [13].

Remark 3.1. Consider a general risk functional $\rho: \mathcal{X} \rightarrow(-\infty, \infty]$ as in Definition 2.3 and the corresponding family $\left(\rho_{r}\right)$ of convex risk measures in (7). Typically each $\rho_{r}$ will admit a robust representation (9) with minimal penalty function $\alpha^{\min }(\cdot, r)$. These representations yield via (8) the following representation of the risk functional $\rho$ :

$$
\begin{aligned}
\rho(X) & =\inf \left\{r \mid E_{Q}[-X] \leq \alpha^{\min }(Q, r) \text { for all } Q \in \mathcal{M}_{1}\right\} \\
& =\sup _{Q \in \mathcal{M}_{1}} r\left(Q, E_{Q}[-X]\right),
\end{aligned}
$$

where $s \mapsto r(Q, s):=\inf \left\{r \in \mathbb{R} \mid s \leq \alpha^{\min }(Q, r)\right\}$ denotes the left inverse of the increasing function $\alpha^{\min }(Q, \cdot)$. We refer to [31] and [32] for a detailed discussion and a large number of examples.

\section{$3.1 \mathcal{X}=L^{\infty}(\Omega, \mathcal{F})$}

Let us denote by $L^{\infty}(\Omega, \mathcal{F})$ the Banach space of all bounded measurable functions on $(\Omega, \mathcal{F})$ and by $\mathcal{M}_{1, f}$ the class of all finitely additive set functions $Q: \mathcal{F} \rightarrow[0,1]$ with $Q[\Omega]=1$. A convex risk measure $\rho$ is Lipschitz continuous with respect to the supremum norm. Applying the FenchelMoreau theorem, combined with the monetary properties of $\rho$ and the weak compactness of $\mathcal{M}_{1, f}$, we obtain the representation

$$
\rho(X)=\max _{Q \in \mathcal{M}_{1, f}}\left\{E_{Q}[-X]-\alpha^{\min }(Q)\right\},
$$

where $\alpha^{\min }(Q)$ is defined as in 107 for any $Q \in \mathcal{M}_{1, f}$, cf. 42, Theorem 4.16. The representation (11) reduces to a robust representation (9) in terms of $\sigma$-additive probability measures, and with max instead of sup, as soon as $\alpha^{\min }(Q)=\infty$ for any $Q \in \mathcal{M}_{1, f} \cap \mathcal{M}_{1}^{c}$. This condition is satisfied iff $\rho$ is continuous from below in the sense that

$$
X_{n} \nearrow X \quad \text { pointwise on } \Omega \quad \Longrightarrow \quad \rho\left(X_{n}\right) \searrow \rho(X) \text {; }
$$

cf., e. g., [42], Theorem 4.22 .

\section{$3.2 \mathcal{X}=C_{b}(\Omega)$}

Here we assume that $\Omega$ is a separable metric space and that $\mathcal{F}$ is the $\sigma$-field of Borel sets. Consider a convex risk measure $\rho$ on $L^{\infty}(\Omega, \mathcal{F})$, and assume that $\rho$ is tight in the sense that there exists an increasing sequence of compact sets $K_{n}$ such that

$$
\lim _{n \uparrow \infty} \rho\left(\lambda 1_{K_{n}}\right)=\rho(\lambda) \quad \text { for any } \lambda \geq 1 .
$$


Then $\rho$, now viewed as a convex risk measure on the space $\mathcal{X}=C_{b}(\Omega)$ of bounded continuous functions on $\Omega$, has a robust representation of the form

$$
\rho(X)=\max _{Q \in \mathcal{M}_{1}}\left\{E_{Q}[-X]-\alpha(Q)\right\} \quad \text { for any } X \in C_{b}(\Omega),
$$

where

$$
\alpha(Q):=\inf \left\{\alpha^{\min }(\tilde{Q}) \mid \tilde{Q} \in \mathcal{M}_{1, f}, E_{\tilde{Q}}[\cdot]=E_{Q}[\cdot] \quad \text { on } C_{b}(\Omega)\right\} .
$$

Moreover, if $\Omega$ is a polish space, then the level sets $\left\{Q \in \mathcal{M}_{1} \mid \alpha(Q) \leq c\right\}$ are relatively compact for the weak topology on $\mathcal{M}_{1}$; cf. [42, Propositions 4.27 and 4.30 .

\section{$3.3 \mathcal{X}=L^{\infty}(\Omega, \mathcal{F}, P)$}

We now fix a probability measure $P$ on $(\Omega, \mathcal{F})$ and denote by $\mathcal{M}_{1}(P)$ the class of all probability measures $Q \in \mathcal{M}_{1}$ which are absolutely continuous with respect to $P$. Let $\rho$ be a convex risk measure on $L^{\infty}(\Omega, \mathcal{F})$ which respects the null sets of $P$, i. e., $\rho(X)=\rho(Y)$ whenever the equivalence relation $X=Y P$-a.s. holds. Then $\rho$ can be regarded as a convex risk measure on the Banach space $\mathcal{X}=L^{\infty}(\Omega, \mathcal{F}, P)$ of equivalence classes. In this case, the robust representation

$$
\rho(X)=\sup _{Q \in \mathcal{M}_{1}(P)}\left\{E_{Q}[-X]-\alpha(Q)\right\}
$$

holds iff $\rho$ is continuous from above, i. e.,

$$
\rho\left(X_{n}\right) \nearrow \rho(X) \quad \text { whenever } X_{n} \searrow X P \text {-a.s. }
$$

or, equivalently, iff $\rho$ has the Fatou property

$$
\rho(X) \leq \liminf _{n \uparrow \infty} \rho\left(X_{n}\right)
$$

for any bounded sequence $\left(X_{n}\right)_{n \in \mathbb{N}}$ in $L^{\infty}(\Omega, \mathcal{F}, P)$ which converges $P$-a.s. to $X$. Moreover, the supremum in $(12)$ is attained for each $X \in L^{\infty}(\Omega, \mathcal{F}, P)$ iff $\rho$ is continuous from below; cf. Delbaen [26] and [42], Theorem 4.33 and Corollary 4.35.

\section{$3.4 \mathcal{X}=L^{p}(\Omega, \mathcal{F}, P)$}

In many practical applications one wants to deal with unbounded random variables, and it is thus natural to consider risk measures on spaces which are larger than $L^{\infty}(\Omega, \mathcal{F}, P)$. Canonical choices are the Banach spaces $L^{p}(\Omega, \mathcal{F}, P)$ with $1 \leq p<\infty$. For a convex risk measure $\rho$ on $\mathcal{X}=L^{p}(\Omega, \mathcal{F}, P)$ the existence of a robust representation is equivalent to lower-semicontinuity of $\rho$ with respect to the $L^{p}$-norm. In this case the robust representation takes the form

$$
\rho(X)=\sup _{Q \in \mathcal{M}^{q}(P)}\left\{E_{Q}[-X]-\alpha^{\min }(Q)\right\}, \quad X \in L^{p}(\Omega, \mathcal{F}, P),
$$

with dual exponent $q:=p /(p-1) \in(1, \infty]$, where we use the notation

$$
\mathcal{M}^{q}(P):=\left\{Q \in \mathcal{M}_{1}(P) \mid \frac{d Q}{d P} \in L^{q}(\Omega, \mathcal{F}, P)\right\} ;
$$

cf. Kaina \& Rüschendorf [63], Theorem 4.6. Moreover, if $\rho$ is a finite convex risk measure on $L^{p}(\Omega, \mathcal{F}, P)$, then it is even Lipschitz continuous with respect to the $L^{p}$-norm, and the representation (13) holds with max instead of sup. For a systematic discussion of risk measures on $L^{p}(\Omega, \mathcal{F}, P)$ we refer to [63] and Filipović \& Svindland [35] and the references therein. 


\subsection{Risk measures on Orlicz hearts}

As a further useful extension beyond the case of bounded random variables, let us consider convex risk measures on Orlicz hearts, as studied by Cheridito \& Li [19, 20]. Let $h:[0, \infty) \rightarrow[0, \infty]$ be a Young function, i. e., a left-continuous and convex function such that $\lim _{x \downarrow 0} h(x)=0$ and $\lim _{x \uparrow \infty} h(x)=\infty$. The conjugate function $h^{*}(y):=\sup _{x \geq 0}\{x y-h(x)\}, y \geq 0$, is again a Young function, and its conjugate is given by $h$. Recall that the Orlicz norm $\|\cdot\|_{h}$ is defined by

$$
\|X\|_{h}:=\inf \left\{a>0 \mid E_{P}\left[h\left(\frac{|X|}{a}\right)\right] \leq 1\right\} .
$$

We denote by $L^{h}(P)$ the corresponding Orlicz space of all random variables $X$ such that $\|X\|_{h}<\infty$. The Orlicz norm $\|\cdot\|_{h^{*}}$ and the Orlicz space $L^{h^{*}}(P)$ are defined in the same way, and this yields the inequality

$$
E_{P}[|X Y|] \leq 2\|X\|_{h}\|Y\|_{h^{*}}
$$

see, e.g., Neveu 69, Appendix A.2.

The convex subset

$$
\mathcal{O}^{h}:=\left\{X \in L^{h}(P) \mid E_{P}[h(c|X|)]<\infty \text { for all } c>0\right\}
$$

of the Orlicz space $L^{h}(P)$ is called the Orlicz heart corresponding to $h$. Any convex risk measure $\rho: \mathcal{O}^{h} \rightarrow \mathbb{R}$ admits the robust representation

$$
\rho(X)=\max _{Q \in \mathcal{M}^{h^{*}}(P)}\left\{E_{Q}[-X]-\alpha^{\min }(Q)\right\}, \quad X \in \mathcal{O}^{h},
$$

in terms of the set

$$
\mathcal{M}^{h^{*}}(P):=\left\{Q \in \mathcal{M}_{1}(P) \mid \frac{d Q}{d P} \in L^{h^{*}}(P)\right\}
$$

cf. [20], Theorem 4.6.

For $h(x)=x^{p}$ with $p \in[1, \infty)$, the Orlicz heart $\mathcal{O}^{h}$ coincides with $L^{p}(\Omega, \mathcal{F}, P)$, and we have $L^{h^{*}}(P)=L^{q}(\Omega, \mathcal{F}, P)$ for the dual exponent $q=p /(p-1)$. Thus, the robust representation of a finite convex risk measure on $L^{p}(\Omega, \mathcal{F}, P)$ can be seen as a special case of the robust representation (17) on Orlicz hearts.

As a further example take the Young function $h(x)=e^{x}-1$. Then the conjugate function $h^{*}$ is given by $h^{*}(y)=(y \log y-y+1) 1_{[1, \infty)}(y)$, and the Orlicz hearts corresponding to the pair $h$ and $h^{*}$ satisfy

$$
\mathcal{O}^{h}=\left\{X \mid E_{P}\left[e^{c|X|}\right]<\infty \text { for all } c>0\right\}, \quad \mathcal{O}^{h^{*}}=\left\{X \mid E_{P}[|X| \log |X|]<\infty\right\} .
$$

The three families of entropic risk measures in Subsection 4.6 below will all be defined on the Orlicz heart $\mathcal{O}^{h}$ in 18 .

\section{Examples}

In the following examples we fix a probability measure $P$ on $(\Omega, \mathcal{F})$. The risk measures will first be considered on the space $\mathcal{X}=L^{\infty}(\Omega, \mathcal{F}, P)$, but typically they will admit a canonical extension to some larger space.

\subsection{Value at Risk}

The most commonly used risk measure in practice is Value at Risk. For a given level $\lambda \in(0,1)$ we denote by $\mathrm{VaR}_{\lambda}$ the monetary risk measure defined by the acceptance set

$$
\mathcal{A}=\{X \in \mathcal{X} \mid P[X<0] \leq \lambda\}
$$


For a financial position $X$, the value $\operatorname{VaR}_{\lambda}(X)$ specifies the smallest monetary amount that needs to be added to $X$ so that the probability of a loss becomes smaller than $\lambda$ :

$$
\begin{aligned}
\operatorname{VaR}_{\lambda}(X) & =\inf \{m \in \mathbb{R} \mid P[X+m<0] \leq \lambda\} \\
& =-\sup \{c \in \mathbb{R} \mid P[X<c] \leq \lambda\}=-q_{X}^{+}(\lambda),
\end{aligned}
$$

where $q_{X}^{+}(\lambda)$ is the upper $\lambda$-quantile of $X$. Clearly, $\operatorname{VaR}_{\lambda}$ is well-defined as a positively homogeneous monetary risk measure on the space $L^{0}(\Omega, \mathcal{F}, P)$ of all finite valued random variables on $(\Omega, \mathcal{F}, P)$. In particular, if $X$ is Gaussian with variance $\sigma_{P}^{2}(X)$, then we have

$$
\operatorname{VaR}_{\lambda}(X)=E_{P}[-X]+\Phi^{-1}(1-\lambda) \sigma_{P}(X),
$$

where $\Phi^{-1}$ denotes the inverse of the distribution function $\Phi$ of the standard normal distribution. Since $\sigma_{P}^{2}$ is a convex functional on $L^{2}(\Omega, \mathcal{F}, P), \operatorname{VaR}_{\lambda}$ can be viewed as a convex risk measure on any Gaussian subspace of $L^{2}(\Omega, \mathcal{F}, P)$ as soon as $\lambda \leq 0.5$. But $\operatorname{VaR}_{\lambda}$ is not convex on $L^{\infty}(\Omega, \mathcal{F}, P)$ and may thus penalize diversification if positions are no longer Gaussian. Note also that $\operatorname{VaR}_{\lambda}$ neglects extreme losses that occur with small probability.

These deficiencies of Value at Risk were a major reason to develop a systematic theory of coherent and convex risk measures, as initiated by [7]. Further drawbacks such as procyclical effects and excessive reliance on a single model $P$ derived from past observations are discussed in The Turner Review - A regulatory response to the global banking crisis [1]. The last point is adressed in the Revisions to the Basel II market risk framework [2] by considering a "Stressed Value at Risk", which involves alternative models $\tilde{P}$ derived from observations and simulations of periods of significant financial stress; cf. also Section 6 below.

\subsection{Average Value at Risk}

Another basic example is the Average Value at Risk, also known as Conditional Value at Risk, Tail Value at Risk, or Expected Shortfall. The Average Value at Risk at level $\lambda \in(0,1]$ is defined by

$$
\operatorname{AVaR}_{\lambda}(X):=\frac{1}{\lambda} \int_{0}^{\lambda} \operatorname{VaR}_{\alpha}(X) d \alpha .
$$

Since $\operatorname{VaR}_{\alpha}$ is increasing in $\alpha$ we have

$$
\operatorname{AVaR}_{\lambda}(X) \geq \operatorname{VaR}_{\lambda}(X) ;
$$

in fact $\mathrm{AVaR}_{\lambda}$ can be characterized as the smallest convex risk measure which is law-invariant in the sense of Section 5 and dominates $\operatorname{VaR}_{\lambda}$.

For $\lambda=1$ it reduces to the expected loss $E_{P}[-X]$, and for $\lambda=0$ we define

$$
\operatorname{AVaR}_{0}(X):=\operatorname{VaR}_{0}(X):=\lim _{\lambda \downarrow 0} \operatorname{VaR}_{\lambda}(X)=\operatorname{ess} \sup (-X) .
$$

For any $\lambda \in(0,1], \mathrm{AVaR}_{\lambda}$ is a coherent risk measure whose robust representation takes the form

$$
\operatorname{AVaR}_{\lambda}(X)=\max _{Q \in \mathcal{Q}_{\lambda}} E_{Q}[-X]
$$

with $\mathcal{Q}_{\lambda}:=\left\{Q \ll P \mid \frac{d Q}{d P} \leq \frac{1}{\lambda}\right\}$; cf., e.g., [42, Theorem 4.52. Moreover, it can be written as

$$
\operatorname{AVaR}_{\lambda}(X)=\frac{1}{\lambda} E_{P}\left[\left(q_{X}(\lambda)-X\right)^{+}\right]-q_{X}(\lambda)=\frac{1}{\lambda} \inf _{z \in \mathbb{R}}\left\{E_{P}\left[(z-X)^{+}\right]-\lambda z\right\}
$$

for any $\lambda$-quantile $q_{X}(\lambda)$ of $X$; cf., e.g., 42, Lemma 4.51. Note also that $\mathrm{AVaR}_{\lambda}$ is well-defined as a coherent risk measure on the space $L^{1}(\Omega, \mathcal{F}, P)$; cf. 35.

Average Value at Risk plays a prominent role in the Swiss Solvency Test; for a careful analysis see Filipović \& Vogelpoth [36]. From a theoretical point of view, Average Value at Risk plays a fundamental role in the context of law-invariance, since it provides the building blocks for any law-invariant convex risk measure; this will be explained in Section 5 


\subsection{Shortfall risk}

Let us define the shortfall risk of a position $X$ as $E_{P}[l(-X)]$, where $l: \mathbb{R} \rightarrow \mathbb{R}$ is a given convex and increasing loss function. In the special case $l(x)=x^{+}$we recover the classical actuarial definition of mean risk ("mittleres Risiko") $E_{P}\left[X^{-}\right]$as discussed, e. g., in Hattendorff [55]. Note that shortfall risk is a risk functional in the sense of Definition 2.3.

Using this notion of shortfall risk, we can now fix a threshold $r_{0}>$ inf $l$ and define the acceptance set

$$
\mathcal{A}:=\left\{X \in \mathcal{X} \mid E_{P}[l(-X)] \leq r_{0}\right\}
$$

$\mathcal{A}$ is clearly convex, and the convex risk measure $\rho^{\mathrm{SR}}$ corresponding to $\mathcal{A}$ via $\sqrt{5}$ is called shortfall risk measure. Since the acceptance set can be rewritten as

$$
\mathcal{A}=\left\{X \in \mathcal{X} \mid E_{P}[u(X)] \geq-r_{0}\right\}
$$

in terms of the utility function $u(x):=-l(-x)$, it is also called utility-based shortfall risk measure.

Note that $m=\rho^{\mathrm{SR}}(X)$ is the unique solution to the equation

$$
E_{P}[l(-X-m)]=r_{0},
$$

and so stochastic root finding techniques can be used for its numerical evaluation; see, e.g., Dunkel \& Weber 34 .

A shortfall risk measure admits a robust representation 12 with minimal penalty function

$$
\alpha^{\min }(Q)=\inf _{\lambda>0} \frac{1}{\lambda}\left(r_{0}+E_{P}\left[l^{*}\left(\lambda \frac{d Q}{d P}\right)\right]\right),
$$

where $l^{*}$ denotes the Fenchel-Legendre transform of $l$. In the special case of a power loss function $l(x)=\frac{1}{p} x^{p} 1_{(0, \infty)}(x)$ with $p \geq 1$, the penalty function is given by

$$
\alpha^{\min }(Q)=\left(p r_{0}\right)^{\frac{1}{p}}\left\|\frac{d Q}{d P}\right\|_{q}
$$

in terms of the dual exponent $q=p /(p-1)$; cf. [41] and [42, Theorem 4.115 and Example 4.118.

\subsection{Divergence risk measures}

For a lower semicontinuous convex function $g: \mathbb{R}_{+} \rightarrow \mathbb{R} \cup\{\infty\}$ with $g(1)<\infty$ and superlinear growth $g(x) / x \rightarrow \infty$ as $x \uparrow \infty$, the corresponding $g$-divergence of a probability measure $Q \in \mathcal{M}_{1}$ with respect to $P$ is defined by

$$
D_{g}(Q \mid P):=E_{P}\left[g\left(\frac{d Q}{d P}\right)\right]
$$

if $Q \ll P$ and by $D_{g}(Q \mid P)=\infty$ otherwise. By Jensen's inequality we have $D_{g}(Q \mid P) \geq D_{g}(P \mid P)=$ $g(1)$. If $P$ is viewed as a benchmark model then it is plausible to use the $g$-divergence with respect to $P$ as a penalty function. The resulting convex risk measure $\rho_{g}$ defined by

$$
\rho_{g}(X)=\sup _{Q \in \mathcal{M}_{1}(P)}\left\{E_{Q}[-X]-D_{g}(Q \mid P)\right\}
$$

is called divergence risk measure. Denoting by $g^{*}(y):=\sup _{x>0}\{x y-g(x)\}$ the convex conjugate function of $g$, the risk measure $\rho_{g}$ can also be represented by the variational identity

$$
\rho_{g}(X)=\inf _{y \in \mathbb{R}}\left\{E_{P}\left[g^{*}(y-X)\right]-y\right\}
$$

cf., e.g., [42], Theorem 4.122 .

Let us now describe some situations where divergence risk measures arise in a natural manner: 
- For $\lambda \in(0,1]$ and the function $g$ defined by $g(x)=0$ for $x \leq \lambda^{-1}$ and $g(x)=\infty$ otherwise, the divergence risk measure $\rho_{g}$ coincides with Average Value at Risk at level $\lambda$. Here we have $g^{*}(y)=\frac{1}{\lambda} y 1_{(0, \infty)}(y)$, and so the variational identity 26 coincides with formula 24p.

- For $g(x)=x \log x$, the $g$-divergence reduces to the relative entropy $H(Q \mid P)$ of $Q$ with respect to $P$, and $\rho_{g}$ is the convex entropic risk measure; see Subsection 4.6.1

- Inserting the minimal penalty function $(25)$ into the robust representation $\sqrt{12}$, the shortfall risk measure $\rho^{\mathrm{SR}}$ with loss function $l$ and threshold $x_{0}$ can be rewritten as

$$
\begin{aligned}
\rho^{\mathrm{SR}}(X) & =\sup _{Q \in \mathcal{M}_{1}(P)}\left\{E_{Q}[-X]-\inf _{\lambda>0}\left\{x_{0}+E_{P}\left[l^{*}\left(\lambda \frac{d Q}{d P}\right)\right]\right\}\right\} \\
& =\sup _{\lambda>0} \sup _{Q \in \mathcal{M}_{1}(P)}\left\{E_{Q}[-X]-E_{P}\left[g_{\lambda}\left(\frac{d Q}{d P}\right)\right]\right\}
\end{aligned}
$$

in terms of the convex functions $g_{\lambda}(y):=\frac{1}{\lambda}\left(l^{*}(\lambda y)+x_{0}\right)$. Thus, the shortfall risk measure can be described as the supremum of certain divergence risk measures, namely

$$
\rho^{\mathrm{SR}}(X)=\sup _{\lambda>0} \rho_{g_{\lambda}}(X) .
$$

- For a utility function $u: \mathbb{R} \rightarrow \mathbb{R} \cup\{-\infty\}$ with $u(0)=0$, Ben-Tal \& Teboulle [11, 12] introduced the optimized certainty equivalent of a financial position $X \in \mathcal{X}$, defined as

$$
S_{u}(X):=\sup _{\eta \in \mathbb{R}}\left\{\eta+E_{P}[u(X-\eta)]\right\} .
$$

This can be interpreted as the present value of an optimal allocation of the uncertain future income $X$ between a certain present amount $\eta$ and the uncertain future amount $X-\eta$. Denote by $g(z):=\sup _{x \in \mathbb{R}}\{x z-l(x)\}$ the convex conjugate function of the loss function $l$ associated to $u$ via $l(x)=-u(-x)$. Then the variational identity (26) yields

$$
S_{u}(X)=-\rho_{g}(X),
$$

i. e., the optimized certainty equivalent coincides, up to a change of sign, with the divergence risk measure $\rho_{g}$.

\subsection{Haezendonck risk measures}

Let $h$ be a Young function as defined in Subsection 3.5. The corresponding Haezendonck risk measure on the Orlicz heart $\mathcal{O}^{h}$ is defined by

$$
\rho(X):=\inf _{z \in \mathbb{R}}\left\{\left\|(z-X)^{+}\right\|_{h}-z\right\}
$$

in terms of the Orlicz norm $\|\cdot\|_{h}$. Then $\rho$ is a coherent risk measure, and its robust representation is given by

$$
\rho(X)=\max _{Q \in \mathcal{M}_{h}} E_{Q}[-X]
$$

where

$$
\mathcal{M}_{h}:=\left\{Q \ll P \mid E_{Q}[Y] \leq\|Y\|_{h} \text { for all } Y \in L_{+}^{\infty}(\Omega, \mathcal{F}, P)\right\} ;
$$

cf. Bellini \& Rosazza Gianin [9], Proposition 17.

In the special case $h(x)=\frac{1}{\lambda} x$ with $\lambda \in(0,1]$, the class $\mathcal{M}_{h}$ coincides with the class $\mathcal{Q}_{\lambda}$ in Subsection 4.2, and so we recover Average Value at Risk at level $\lambda$ as a special case. In particular, definition (27) reduces to the representation (24) of $\mathrm{AVaR}_{\lambda}$. 


\subsection{Entropic risk measures}

In this subsection we focus on three families of monetary risk measures which are defined in terms of the relative entropy

$$
H(Q \mid P):=\left\{\begin{array}{cl}
E_{Q}\left[\log \frac{d Q}{d P}\right] & \text { if } Q \ll P, \\
+\infty & \text { otherwise. }
\end{array}\right.
$$

As mentioned in Subsection 4.4 the relative entropy can be seen as a $g$-divergence for the function $g(x)=x \log x$.

Note that the following construction of convex, coherent, and truncated risk measures also applies if relative entropy is replaced by a general penalty function $\alpha$.

\subsubsection{The convex entropic risk measure}

Let us first consider the case where the penalty function is of the form

$$
\alpha(Q):=\frac{1}{\gamma} H(Q \mid P)
$$

with some constant $\gamma>0$.

Definition 4.1. The convex risk measure $e_{\gamma}$ defined by

$$
e_{\gamma}(X):=\sup _{Q \in \mathcal{M}_{1}}\left\{E_{Q}[-X]-\frac{1}{\gamma} H(Q \mid P)\right\}
$$

is called the (convex) entropic risk measure with parameter $\gamma$.

Using the well-known variational principle

$$
H(Q \mid P)=\sup _{X \in L^{\infty}(\Omega, \mathcal{F}, P)}\left\{E_{Q}[-X]-\log E_{P}\left[e^{-X}\right]\right\}
$$

for the relative entropy, it follows that $e_{\gamma}$ takes the explicit form

$$
e_{\gamma}(X)=\frac{1}{\gamma} \log E_{P}\left[e^{-\gamma X}\right]
$$

cf., e.g., 42, Example 4.34. Clearly, $e_{\gamma}$ is well-defined as a finite convex risk measure on the Orlicz heart 18 with respect to the function $h(x)=e^{x}-1$.

The acceptance set (4) of $e_{\gamma}$ takes the form

$$
\begin{aligned}
\mathcal{A} & =\left\{X \mid e_{\gamma}(X) \leq 0\right\} \\
& =\left\{X \mid E_{P}\left[l_{\gamma}(-X)\right] \leq 1\right\}=\left\{X \mid E_{P}\left[u_{\gamma}(X)\right] \geq 0\right\}
\end{aligned}
$$

in terms of the convex loss function $l_{\gamma}(x)=e^{\gamma x}$ and the exponential utility function $u_{\gamma}(x)=$ $1-e^{-\gamma x}$, and so the convex entropic risk measure is a special case of the shortfall risk measure in Subsection 4.3 .

It is easy to see that $e_{\gamma}(X)$ is increasing in $\gamma$ and strictly increasing as soon as $X$ is not constant $P$-a.s.. Moreover,

$$
e_{0}(X):=\lim _{\gamma \downarrow 0} e_{\gamma}(X)=E_{P}[-X] \quad \text { and } \quad e_{\infty}(X):=\lim _{\gamma \uparrow \infty} e_{\gamma}(X)=\operatorname{ess} \sup (-X) ;
$$

cf., e.g., Kaas, Goovaerts, Dhaene \& Denuit [62], Theorem 1.3.2.

Formula 28 implies that $-e_{\gamma}$ is a certainty equivalent with respect to the exponential utility function $u_{\gamma}$, i.e.,

$$
u_{\gamma}\left(-e_{\gamma}(X)\right)=E_{P}\left[u_{\gamma}(X)\right]
$$


As shown by de Finetti, convex entropic risk measures can actually be characterized by this property: If $\rho$ is a monetary risk measure and $-\rho$ is a certainty equivalent with respect to some strictly increasing continuous function $u$, then $\rho$ must be a convex entropic risk measure $e_{\gamma}$ for some parameter $\gamma \geq 0$, including the linear case $\gamma=0$; cf. de Finetti [24] or, e. g., Example 4.13 in [42].

For any $\gamma \in[0, \infty]$, the risk measure $e_{\gamma}$ is additive on independent positions, i. e.,

$$
e_{\gamma}(X+Y)=e_{\gamma}(X)+e_{\gamma}(Y)
$$

if $X$ and $Y$ are independent under $P$. Clearly, this additivity is preserved for any risk measure of the form

$$
\rho(X)=\int_{[0, \infty]} e_{\gamma}(X) \nu(d \gamma)
$$

with some probability measure $\nu$ on $[0, \infty]$. For an axiomatic characterization of such mixtures we refer to Goovaerts, Kaas, Laeven \& Tang [52] and Goovaerts \& Laeven [53]; cf. also Gerber \& Goovaerts [47, where an analogous problem is discussed in the context of actuarial premium principles.

Remark 4.1. Consider a portfolio of n positions, given as non-constant random variables $X_{1}, \ldots, X_{n}$ with finite exponential moments which are independent and identically distributed under $P$. The additivity (30) of $e_{\gamma}$ implies

$$
\pi_{n}:=\frac{1}{n} e_{\gamma}\left(X_{1}+\ldots+X_{n}\right)=e_{\gamma}\left(X_{1}\right)>E_{P}\left[-X_{1}\right]
$$

i. e., the capital requirement per position does not decrease to the risk-neutral value $E_{P}\left[-X_{1}\right]$ as the portfolio becomes large.

\subsubsection{The coherent entropic risk measure}

As our second example, we fix some threshold $c>0$ and consider the coherent risk measure defined via (31) by the class of probability measures $Q$ whose deviation from the reference measure $P$, as quantified by the relative entropy $H(Q \mid P)$, does not exceed the level $c$.

Definition 4.2. For each $c>0$, the risk measure $\rho_{c}$ defined by

$$
\rho_{c}(X):=\sup _{Q \in \mathcal{M}_{1}: H(Q \mid P) \leq c} E_{Q}[-X], \quad X \in L^{\infty}(\Omega, \mathcal{F}, P)
$$

will be called the coherent entropic risk measure at level $c$.

The supremum in (31) is actually attained, as shown in Föllmer \& Knispel [38], Proposition 3.1. Indeed, consider the exponential family

$$
\mathcal{Q}_{X, P}=\left\{Q_{X, \gamma} \mid \gamma \in \mathbb{R}\right\}
$$

induced by $P$ and $-X$, where

$$
\frac{d Q_{X, \gamma}}{d P}=e^{-\gamma X}\left(E_{P}\left[e^{-\gamma X}\right]\right)^{-1} .
$$

If $p(X):=P[X=\operatorname{ess} \inf X]>0$ then we also include the limiting measure $Q_{X, \infty}:=\lim _{\gamma \uparrow \infty} Q_{X, \gamma}=$ $P[\cdot \mid X=\operatorname{essinf} X]$.

Proposition 4.1. For $c \in(0,-\log p(X))$ we have

$$
\rho_{c}(X)=\max _{Q \in \mathcal{M}_{1}: H(Q \mid P) \leq c} E_{Q}[-X]=E_{Q_{X, \gamma_{c}}}[-X],
$$

where $Q_{X, \gamma_{c}} \in \mathcal{Q}_{P, X}$ and $\gamma_{c}>0$ is such that $H\left(Q_{X, \gamma_{c}} \mid P\right)=c$, and moreover

$$
\rho_{c}(X)=\min _{\gamma>0}\left\{\frac{c}{\gamma}+e_{\gamma}(X)\right\}=\frac{c}{\gamma_{c}}+e_{\gamma_{c}}(X) .
$$


If $p(X)>0$ and $c \geq-\log p(X)$, then

$$
\rho_{c}(X)=E_{Q_{X, \infty}}[-X]=\operatorname{ess} \sup (-X) .
$$

Proposition 4.1 implies that the coherent risk measure $\rho_{c}$ is continuous from above and even continuous from below; see, e.g., [42, Theorem 4.33 together with Corollary 4.38. It also shows that $\rho_{c}$ is well-defined as a finite coherent risk measure on the Orlicz heart $\mathcal{O}^{h}$ in (18). Moreover, $\rho_{c}$ is increasing in $c$ with

$$
\lim _{c \downarrow 0} \rho_{c}(X)=E_{P}[-X] \quad \text { and } \quad \lim _{c \uparrow \infty} \rho_{c}(X)=\operatorname{ess} \sup (-X) ;
$$

cf. [38, Corollary 3.1.

Note also that $H(Q \mid P) \leq-\log \lambda$ for any $Q$ such that $\frac{d Q}{d P} \leq \frac{1}{\lambda}$, and so the robust representation 23. of Average Value at Risk implies

$$
\operatorname{AVaR}_{\lambda}(X) \leq \rho_{-\log \lambda}(X) .
$$

Remark 4.2. In the situation of Remark 4.1, a coherent entropic risk measure $\rho_{c}$ behaves differently from the convex risk measures $e_{\gamma}$ : The capital requirement

$$
\pi_{n}:=\frac{1}{n} \rho_{c}\left(X_{1}+\ldots+X_{n}\right)
$$

decreases to $E_{P}\left[-X_{1}\right]$ as $n$ tends to infinity, and it does so at the rate $n^{-1 / 2}$. More precisely,

$$
\lim _{n \uparrow \infty} \sqrt{n}\left(\pi_{n}-E_{P}\left[-X_{1}\right]\right)=\sigma_{P}\left(X_{1}\right) \sqrt{2 c},
$$

where $\sigma_{P}^{2}\left(X_{1}\right)$ denotes the variance of $X_{1}$ under $P$; cf. [38], Proposition 4.1.

\subsubsection{The truncated convex entropic risk measure}

Let us finally consider a truncated version of the convex entropic risk measure $e_{\gamma}$ :

Definition 4.3. For parameters $\gamma>0$ and $c>0$, we define the truncated entropic risk measure $e_{\gamma, c} b y$

$$
e_{\gamma, c}(X):=\sup _{Q \in \mathcal{M}_{1}: H(Q \mid P) \leq c}\left\{E_{Q}[-X]-\frac{1}{\gamma} H(Q \mid P)\right\} .
$$

Clearly,

$$
e_{\gamma, c}(X) \leq \min \left\{\rho_{c}(X), e_{\gamma}(X)\right\},
$$

and $e_{\gamma, c}$ is well-defined as a finite convex risk measure on the Orlicz heart $\mathcal{O}^{h}$ in 18p. For a nonconstant position $X \in \mathcal{O}^{h}$, the supremum in 36 is actually attained by the measure $Q_{X, \gamma \wedge \beta(c)}$ in the exponential family $\mathcal{Q}_{X, P}$, where $\beta(c)$ is the unique parameter $\beta>0$ such that $H\left(Q_{X, \beta} \mid P\right)=c$ for $c \in(0,-\log p(X))$ and $\beta(c):=\infty$ otherwise; cf. Föllmer \& Knispel [39], Lemma 4.2.

Remark 4.3. In the situation of Remark 4.2. the capital requirements specified by a truncated entropic risk measure $e_{\gamma, c}$ are clearly lower than the capital requirements prescribed by the coherent entropic risk measure $\rho_{c}$. But their asymptotic behavior is exactly the same as in formula (35); cf. [39], Corollary 4.1. 


\section{Law-invariant convex risk measures}

Let us fix again a probability measure $P$ on $(\Omega, \mathcal{F})$. Throughout this section we assume that the probability space $(\Omega, \mathcal{F}, P)$ is atomless.

Definition 5.1. A monetary risk measure $\rho$ on $L^{\infty}(\Omega, \mathcal{F}, P)$ is called law-invariant if $\rho(X)$ only depends on the distribution of $X$ under $P$, i. e., if $\rho(X)=\rho(Y)$ whenever $X$ and $Y$ have the same distribution under $P$.

Value at Risk as defined in $(19)$ is clearly law-invariant, and the same is true for all the other examples introduced in Subsection 4. Average Value at Risk, shortfall risk measures, divergence risk measures, Haezendonck risk measures, and the entropic risk measures.

Consider a convex risk measure $\rho$ on $L^{\infty}(\Omega, \mathcal{F}, P)$ which is continuous from above and thus admits a robust representation $(12)$ in terms of probability measures $Q \in \mathcal{M}_{1}(P)$ and the minimal penalty function $\alpha^{\min }$. Then $\rho$ is law-invariant if and only if $\alpha^{\min }(Q)$ depends only on the law of the density $\frac{d Q}{d P}$ under $P$; cf., e.g., [42, Theorem 4.59. In fact continuity from above is implied by law-invariance if $L^{2}(\Omega, \mathcal{F}, P)$ is separable; cf. Jouini, Schachermayer \& Touzi [61].

If $\rho$ is a convex risk measure on $L^{\infty}(\Omega, \mathcal{F}, P)$ which is law-invariant, then it admits a canonical extension to a law-invariant convex risk measure on $L^{1}(\Omega, \mathcal{F}, P)$ defined by $(13)$ with $p=1$ and $q=\infty$; cf. 35 .

As shown by Kusuoka [66] in the coherent case and by Kunze [65, Dana [23] and Frittelli \& Rosazza Gianin [45] in the general convex case, any law-invariant convex risk measure $\rho$ can be constructed by using AVaR as building blocks:

Theorem 5.1. A convex risk measure is law-invariant if and only if

$$
\rho(X)=\sup _{\mu \in \mathcal{M}_{1}((0,1])}\left(\int_{(0,1]} \operatorname{AVaR}_{\lambda}(X) \mu(d \lambda)-\beta^{\min }(\mu)\right),
$$

where $\beta^{\text {min }}$ denotes the minimal penalty function given by

$$
\beta^{\min }(\mu)=\sup _{X \in \mathcal{A}_{\rho}} \int_{(0,1]} \operatorname{AVaR}_{\lambda}(X) \mu(d \lambda) .
$$

We refer to [42], Theorem 4.62, for the proof, and also to Drapeau, Kupper \& Reda [33] for an extension of the representation theorem to law-invariant risk functionals in the sense of Definition 2.3 .

In the coherent case we have $\beta^{\text {min }} \in\{0, \infty\}$. Thus a coherent risk measure $\rho$ is law-invariant if and only if

$$
\rho(X)=\sup _{\mu \in \mathcal{M}} \int_{(0,1]} \operatorname{AVaR}_{\lambda}(X) \mu(d \lambda)
$$

for some set $\mathcal{M} \subset \mathcal{M}_{1}((0,1])$. Note that $\rho$ reduces to the linear case $\rho(X)=E_{P}[-X]$ if and only if the representing set in 38 reduces to $\mathcal{M}=\left\{\delta_{1}\right\}$. In any other case $\rho$ will charge a risk premium on top of the expected loss $E_{P}[-X]$ :

Corollary 5.1. Let $\rho$ be a law-invariant coherent risk measure with $\mathcal{M} \neq\left\{\delta_{1}\right\}$. Then

$$
\rho(X)>E_{P}[-X]
$$

for any non-constant position $X \in L^{1}(\Omega, \mathcal{F}, P)$. In particular,

$$
0 \neq X \in \mathcal{A}_{\rho} \quad \Rightarrow \quad E_{P}[-X]>0 \text {. }
$$


Proof. For a non-constant $X$ we have

$$
\operatorname{AVaR}_{\lambda}(X)=-\frac{1}{\lambda} \int_{0}^{\lambda} q_{X}(\alpha) d \alpha>-\int_{0}^{1} q_{X}(\alpha) d \alpha=E_{P}[-X]
$$

for any $\lambda \in(0,1)$, hence

$$
\rho(X) \geq \int_{(0,1]} \operatorname{AVaR}_{\lambda}(X) \mu(d \lambda)>E_{P}[-X]
$$

for any $\mu \in \mathcal{M}$ such that $\mu[\{1\}]<1$. This shows $[39$, and in particular 40 .

Example 5.1. For the coherent entropic risk measure $\rho_{c}$ defined by (31) the representing class $\mathcal{M}_{c}$ takes the form

$$
\mathcal{M}_{c}=\left\{\mu \in \mathcal{M}_{1}((0,1]) \mid \int_{0}^{1} q_{\mu}(t) \log q_{\mu}(t) d t \leq c\right\},
$$

where $q_{\mu}$ denotes the function on $(0,1)$ associated to $\mu \in \mathcal{M}_{1}((0,1])$ via

$$
q_{\mu}(t):=\int_{(1-t, 1]} \frac{1}{s} \mu(d s)
$$

cf. [39], Proposition 4.2.

\subsection{Choquet integrals and concave distortions}

Let us focus on the special class of law-invariant risk measures which can be represented as mixtures of Average Value at Risk, i. e.,

$$
\rho_{\mu}(X):=\int_{[0,1]} \operatorname{AVaR}_{\lambda}(X) \mu(d \lambda), \quad \mu \in \mathcal{M}_{1}([0,1]) .
$$

Such a risk measure $\rho_{\mu}$ is coherent, and it can be rewritten as the Choquet integral

$$
\rho_{\mu}(X)=\int(-X) d c:=\int_{0}^{\infty} c[-X>x] d x+\int_{-\infty}^{0}(c[-X>x]-1) d x
$$

with respect to the concave distortion $c=\psi \circ P$ of $P$, where $\psi$ denotes the increasing and concave function on the unit interval defined by $\psi(0)=0$ and the right-hand derivative

$$
\psi_{+}^{\prime}(t)=\int_{(t, 1]} s^{-1} \mu(d s), \quad 0<t<1 ;
$$

cf., e. g., [42], Theorem 4.70 .

Conversely, for any increasing concave function $\psi$ on $[0,1]$ with $\psi(0)=0$ and $\psi(1)=1$ there is a unique probability measure $\mu$ on $[0,1]$ such that $\psi$ is given by [45); cf., e.g., [42, Lemma 4.69. Thus the Choquet integral $\int(-X) d c$ with respect to any concave distortion $c=\psi \circ P$ can be represented in the form (43) as a mixture of the values $\operatorname{AVaR}_{\lambda}(X)$.

Example 5.2. For a positive integer $n$ consider the concave distortion $c=\psi \circ P$, where $\psi(x)=$ $1-(1-x)^{n}$. The corresponding risk measure $\rho$, defined as the Choquet integral of the loss as in (44), is sometimes called MINVAR. For independent copies $X_{1}, \ldots, X_{n}$ of $X$ we get

$$
\rho(X)=E_{P}\left[\max \left\{-X_{1}, \ldots,-X_{n}\right\}\right]=-E_{P}\left[\min \left\{X_{1}, \ldots, X_{n}\right\}\right],
$$

i. e., $\rho(X)$ can be described as the expectation under $P$ of the maximal loss occurring in the portfolio $X_{1}, \ldots, X_{n}$. The risk measures MAXVAR, MAXMINVAR and MINMAXVAR are defined in a same manner by the distortion functions $x^{1 / n},\left(1-(1-x)^{n}\right)^{1 / n}$ and $1-\left(1-x^{1 / n}\right)^{n}$ respectively; cf. Cherny \& Madan [21]. 
The risk measure $\rho_{\mu}$ is also comonotonic, i. e., $\rho_{\mu}$ satisfies

$$
\rho_{\mu}(X+Y)=\rho_{\mu}(X)+\rho_{\mu}(Y)
$$

whenever two positions $X$ and $Y$ are comonotone in the sense that

$$
\left(X(\omega)-X\left(\omega^{\prime}\right)\right)\left(Y(\omega)-Y\left(\omega^{\prime}\right)\right) \geq 0 \quad \text { for all }\left(\omega, \omega^{\prime}\right) \in \Omega \times \Omega ;
$$

cf., e.g., 42, Theorem 4.88 combined with Corollary 4.77. Conversely, any law-invariant and comonotonic risk measure can be written in the form 443 for some probability measure on $[0,1]$ or, equivalently, as the Choquet integral (44) of the loss with respect to some concave distortion $c=\psi \circ P$; cf., e. g., 42], Theorem 4.93 or [25, 26].

\subsection{Law-invariance and stochastic dominance}

Law-invariant convex risk measures are compatible with stochastic dominance; this follows from their representation (37) in terms of Average Value at Risk. Conversely, this compatibility yields an alternative characterization of law-invariant convex risk measures in terms of comonotonic convexity, as shown by Song \& Yan [78] and stated in Proposition 5.1 below.

Let us first recall the two standard notions of stochastic dominance. For random variables $X$ and $Y$ in $\mathcal{X}=L^{\infty}(\Omega, \mathcal{F}, P)$ with distributions $\mu_{X}$ and $\mu_{Y}$, distribution functions $F_{X}$ and $F_{Y}$, and quantile functions $q_{X}$ and $q_{Y}$, consider the two partial orders

$$
\begin{aligned}
X \succeq_{(1)} Y & : \Longleftrightarrow F_{X}(x) \leq F_{Y}(x) \quad \text { for all } x \in \mathbb{R} \\
& \Longleftrightarrow q_{X}(\alpha) \geq q_{Y}(\alpha) \quad \text { for all } \alpha \in(0,1)
\end{aligned}
$$

and

$$
\begin{aligned}
X \succeq_{(2)} Y & : \Longleftrightarrow \int_{-\infty}^{x} F_{X}(z) d z \leq \int_{-\infty}^{x} F_{Y}(z) d z \quad \text { for all } x \in \mathbb{R} \\
& \Longleftrightarrow \int_{0}^{\lambda} q_{X}(\alpha) d \alpha \geq \int_{0}^{\lambda} q_{Y}(\alpha) d \alpha \quad \text { for all } \lambda \in(0,1),
\end{aligned}
$$

often called stochastic dominance of the first and the second kind. Equivalently we can write

$$
X \succeq_{(i)} Y \quad: \Longleftrightarrow \int u d \mu_{X} \geq \int u d \mu_{Y} \quad \text { for all } u \in \mathcal{U}^{(i)},
$$

where $\mathcal{U}^{(1)}\left(\right.$ resp. $\left.\mathcal{U}^{(2)}\right)$ denotes the class of all increasing (resp. all increasing and concave) functions $u: \mathbb{R} \rightarrow \mathbb{R}$, and also

$$
X \succeq_{(2)} Y \quad \Longleftrightarrow \quad E_{P}\left[(c-X)^{+}\right] \leq E_{P}\left[(c-Y)^{+}\right] \quad \text { for any } c \in \mathbb{R} .
$$

A monetary risk measure $\rho$ on $\mathcal{X}$ is law-invariant if and only if it respects stochastic dominance of the first kind, i. e.,

$$
X \succeq_{(1)} Y \quad \Longrightarrow \quad \rho(X) \leq \rho(Y) .
$$

Indeed, since $(\Omega, \mathcal{F}, P)$ is assumed to be atomless, there exists a random variable $U$ with uniform distribution on $(0,1)$, and law-invariance together with monotonicity of the monetary risk measure $\rho$ implies

$$
\rho(X)=\rho\left(q_{X}(U)\right) \leq \rho\left(q_{Y}(U)\right)=\rho(Y),
$$

since $q_{X} \geq q_{Y}$ if $X \succeq_{(1)} Y$. Conversely, 49 implies $\rho(X)=\rho(Y)$ whenever $X$ and $Y$ have the same distribution. 
As to stochastic dominance of the second kind, note first that the second part of (47) translates into the equivalence

$$
X \succeq_{(2)} Y \quad \Longleftrightarrow \quad \operatorname{AVaR}_{\lambda}(X) \leq \operatorname{AVaR}_{\lambda}(Y) \text { for all } \lambda \in(0,1),
$$

since $\operatorname{VaR}_{\alpha}(X)=-q_{X}(\alpha)$ for almost all $\alpha \in(0,1)$. The representation (37) of law-invariant convex risk measures in terms of Average Value at Risk shows that any convex and law-invariant risk measure $\rho$ respects stochastic dominance of the second kind, i. e.,

$$
X \succeq_{(2)} Y \quad \Longrightarrow \quad \rho(X) \leq \rho(Y) \text {. }
$$

Moreover, we obtain the equivalence

$$
X \succeq_{(2)} Y \Longleftrightarrow \rho(X) \leq \rho(Y) \text { for any } \rho \in \mathcal{R},
$$

where $\mathcal{R}$ can be an arbitrary class of law-invariant convex risk measures that contains $\mathrm{AVaR}_{\lambda}$ for any $\lambda \in(0,1)$. For example we could take the class of comonotonic risk measures $\rho_{\mu}$ in the preceding subsection; cf., e.g., Dhaene, Kukush \& Pupashenko [29] and Dhaene et al. [30].

Conversely, convexity follows from $(50)$ combined with the following property of comonotonic convexity: For $X, Y \in \mathcal{X}$ and $\lambda \in(0,1)$,

$$
\rho(\lambda X+(1-\lambda) Y) \leq \lambda \rho(X)+(1-\lambda) \rho(Y) \text { if } X \text { and } Y \text { are comonotonic; }
$$

78, Theorem 3.6. More precisely:

Proposition 5.1. Let $\rho$ be a monetary risk measure on $\mathcal{X}=L^{\infty}(\Omega, \mathcal{F}, P)$. Then $\rho$ is law-invariant and convex if and only if $\rho$ respects second order stochastic dominance and satisfies the condition (52) of comonotonic convexity.

The "only if" part is already clear. To see the converse, note first that condition (50) implies law-invariance of $\rho$. To check convexity, take $X$ and $Y$ in $\mathcal{X}$ and $\lambda \in(0,1)$, and define $X:=q_{X}(U)$ and $\tilde{Y}:=q_{Y}(U)$, where $U$ is uniformly distributed on $[0,1]$. Since $\lambda \tilde{X}$ and $(1-\lambda) \tilde{Y}$ are comonotone, any comonotonic risk measure $\rho_{\mu}$ in 43 satisfies

$$
\begin{aligned}
\rho_{\mu}(\lambda X+(1-\lambda) Y) & \leq \rho_{\mu}(\lambda X)+\rho_{\mu}((1-\lambda) Y) \\
& =\rho_{\mu}(\lambda \tilde{X})+\rho_{\mu}((1-\lambda) \tilde{Y}) \\
& =\rho_{\mu}(\lambda \tilde{X}+(1-\lambda) \tilde{Y}) .
\end{aligned}
$$

Due to 51, this implies $\lambda X+(1-\lambda) Y \succeq_{(2)} \lambda \tilde{X}+(1-\lambda) \tilde{Y}$. Using first 50 and then comonotonic convexity and law-invariance, we obtain

$$
\begin{aligned}
\rho(\lambda X+(1-\lambda) Y) & \leq \rho(\lambda \tilde{X}+(1-\lambda) \tilde{Y}) \\
& \leq \lambda \rho(\tilde{X})+(1-\lambda) \rho(\tilde{Y}) \\
& =\lambda \rho(X)+(1-\lambda) \rho(Y),
\end{aligned}
$$

and so $\rho$ is indeed a convex risk measure.

In the same way, law-invariant coherent risk measures on $\mathcal{X}$ can be characterized as positively homogeneous monetary risk measures on $\mathcal{X}$ which respect stochastic dominance of the second kind and satisfy the following property of comonotonic subadditivity:

$$
\rho(X+Y) \leq \rho(X)+\rho(Y) \text { if } X \text { and } Y \text { are comonotone; }
$$

cf. 78, Theorem 3.2 . 


\subsection{Risk measures on lotteries}

Consider a law-invariant monetary risk measure $\rho$ on $\mathcal{X}=L^{\infty}(\Omega, \mathcal{F}, P)$. The distribution $\mu_{X}$ of $X \in \mathcal{X}$ under $P$ belongs to the class $\mathcal{M}_{1, c}(\mathbb{R})$ of probability measures on $\mathbb{R}$ with compact support, often called lotteries. Any lottery $\mu \in \mathcal{M}_{1, c}(\mathbb{R})$ arises in this way, since we have assumed that $(\Omega, \mathcal{F}, P)$ is atomless. The risk measure $\rho$ can thus be identified with the functional $R$ : $\mathcal{M}_{1, c}(\mathbb{R}) \rightarrow \mathbb{R}$ on lotteries defined by

$$
R\left(\mu_{X}\right)=\rho(X) .
$$

The monotonicity of $\rho$ amounts to monotonicity of $R$ with respect to stochastic dominance of the first kind, viewed as a partial order on lotteries:

$$
\mu \preceq_{(1)} \nu \quad \Longrightarrow \quad R(\mu) \geq R(\nu) .
$$

Cash-invariance of $\rho$ translates into the condition

$$
R\left(T_{m} \mu\right)=R(\mu)-m,
$$

where $T_{m} \mu$ denotes the shift of $\mu$ by the amount $m$, i. e., $T_{m} \mu(A):=\mu(A-m)$.

\subsubsection{Two notions of convexity and a characterization of shortfall risk measures}

The risk measure $R$ on lotteries will be called convex if the underlying risk measure $\rho$ on $\mathcal{X}$ is convex. In this case $R$ respects stochastic dominance of the second kind, viewed as a partial order on lotteries:

$$
\mu \preceq_{(2)} \nu \quad \Longrightarrow \quad R(\mu) \geq R(\nu)
$$

cf. (50). Note that lotteries can be identified with their quantile functions. At this level, Proposition 5.1 and its proof show that convexity of $R$ can be characterized by two conditions: $R$ satisfies (54), and $R$ is a convex functional on the convex cone of quantile functions.

At the level of lotteries, however, we may also want to require a different type of convexity, namely convexity of the acceptance set

$$
\mathcal{A}_{R}:=\left\{\mu \in \mathcal{M}_{1, c}(\mathbb{R}) \mid R(\mu) \leq 0\right\} .
$$

In other words, if two lotteries $\mu$ and $\nu$ are acceptable, then any compound lottery $\alpha \mu+(1-\alpha) \nu$ obtained by randomizing the choice between $\mu$ and $\nu$ with some probability $\alpha \in(0,1)$ should also be acceptable. This second kind of convexity is indeed satisfied if $\rho$ is a shortfall risk measure as defined in Subsection 4.3. Here the acceptance set

$$
\mathcal{A}_{R}=\left\{\mu \in \mathcal{M}_{1, c}(\mathbb{R}) \mid \int l(-x) \mu(d x) \leq r_{0}\right\}
$$

is clearly convex, and so is its complement $\mathcal{A}_{R}^{c}$. Conversely, as shown by Weber [83] under some mild regularity conditions, a law-invariant monetary risk measure $R$ on $\mathcal{M}_{1, c}(\mathbb{R})$ must be a shortfall risk measure for some increasing loss function $l$ if both the acceptance set $\mathcal{A}_{R}$ and its complement $\mathcal{A}_{R}^{c}$ are convex. In this case, convexity of the loss function $l$ is equivalent to the first kind of convexity of $R$, namely to the convexity of the underlying risk measure $\rho$ on $\mathcal{X}$.

\subsubsection{A characterization of convex entropic risk measures}

Let us derive an alternative characterization of the convex entropic risk measures $e_{\gamma}$. Let $\rho$ be a normalized and law-invariant risk measure on $\mathcal{X}$. For the corresponding risk measure $R$ on lotteries we consider the partial order $\preceq_{R}$ on lotteries defined by

$$
\mu \preceq_{R} \nu \quad: \Longleftrightarrow \quad R(\mu) \geq R(\nu)
$$


for $\mu, \nu \in \mathcal{M}_{1, c}(\mathbb{R})$. If $R$ corresponds to an entropic risk measure $e_{\gamma}$ with some parameter $\gamma \geq 0$ then $\preceq_{R}$ satisfies the von Neumann-Morgenstern axioms for preference orders on lotteries, and in particular the independence axiom:

$$
\mu \preceq_{R} \nu \quad \Longrightarrow \quad \alpha \mu+(1-\alpha) \lambda \preceq_{R} \alpha \nu+(1-\alpha) \lambda
$$

for any lottery $\lambda$ and any $\alpha \in(0,1)$. Let us now show that, conversely, the validity of these axioms for $\preceq_{R}$ implies that $\rho$ is a convex entropic risk measure. Recall first that the axioms of von Neumann-Morgenstern yield a numerical representation in terms of expected utility, i. e.,

$$
\mu \preceq_{R} \nu \quad \Longleftrightarrow \quad \int u d \mu \leq \int u d \nu
$$

for some increasing continuous function $u$ on $\mathbb{R}$; cf., e. g., 42, Corollary 2.28 and Proposition 2.33. By cash-invariance and normalization we have $R\left(\delta_{x}\right)=-x$, hence $\delta_{x} \prec_{R} \delta_{y}$ for $x<y$. Thus $u$ is strictly increasing, and we denote by

$$
C(\mu):=u^{-1}\left(\int u d \mu\right)
$$

the corresponding certainty equivalent on lotteries. Now note that $\delta_{C(\mu)} \sim_{R} \mu$ implies

$$
R(\mu)=R\left(\delta_{C(\mu)}\right)=-C(\mu),
$$

and so $-R$ coincides with the certainty equivalent $C$. Applying de Finetti's criterion 24 as in Subsection 4.6.2 we conclude that $R$, or rather the underlying monetary risk measure $\rho$, is of the form $e_{\gamma}$ with some parameter $\gamma \geq 0$.

\subsection{Risk measures and generalized deviations}

Any law-invariant convex risk measure $\rho$ on $\mathcal{X}=L^{p}(\Omega, \mathcal{F}, P), 1 \leq p \leq \infty$, satisfies

$$
\rho\left(E_{P}[X \mid \mathcal{G}]\right) \leq \rho(X)
$$

for $X \in \mathcal{X}$ and any sub- $\sigma$-field $\mathcal{G} \subseteq \mathcal{F}$. This follows from (51), since Jensen's inequality implies

$$
E_{P}[X \mid \mathcal{G}] \succeq_{(2)} X
$$

If $\rho$ is normalized by $\rho(0)=0$, then we get in particular $\rho(X) \geq E_{P}[-X]$, and typically we have

$$
\rho(X)>E_{P}[-X] \text { for non-constant } X,
$$

as illustrated by the examples in Section 4 and by Corollary 5.1 .

More generally, consider any convex risk measure $\rho$ on $\mathcal{X}$ which satisfies condition (55). As proposed by Rockafellar, Uryasev and Zabarankin in [74], we could now focus on the functional $\mathcal{D}: \mathcal{X} \rightarrow[0, \infty]$ defined by

$$
\mathcal{D}(X):=\rho(X)-E_{P}[-X] .
$$

Clearly, $\mathcal{D}$ inherits from $\rho$ the following properties:

i) $\mathcal{D}(X) \geq 0$ for $X \in \mathcal{X}$, with $\mathcal{D}(X)>0$ for non-constant $X$,

ii) $\mathcal{D}(X+m)=\mathcal{D}(X)$ for $X \in \mathcal{X}$ and $m \in \mathbb{R}$,

iii) $\mathcal{D}$ is convex on $\mathcal{X}$.

If $\rho$ is coherent then $\mathcal{D}$ is also positively homogeneous, i. e., 
iv) $\mathcal{D}(\lambda X)=\lambda \mathcal{D}(X)$ for $X \in \mathcal{X}$ and $\lambda \geq 0$,

and iii) is equivalent to subadditivity, i. e.,

v) $\mathcal{D}(X+Y) \leq \mathcal{D}(X)+\mathcal{D}(Y)$ for $X, Y \in \mathcal{X}$.

Moreover, the robust representation (13) of a coherent risk measure $\rho$ on $L^{p}(\Omega, \mathcal{F}, P)$ translates into a robust representation of $\mathcal{D}$. In the special case $p=2$ this takes the form

$$
\mathcal{D}(X)=E_{P}[X]-\inf _{Z \in \mathcal{Z}} E_{P}[X Z]
$$

for some closed and convex subset $\mathcal{Z}$ of $L^{2}(\Omega, \mathcal{F}, P)$ such that $E_{P}[Z]=1$ for all $Z \in \mathcal{Z}$, and for any non-constant $X$ we have $E_{P}[X Z]<E_{P}[X]$ for some $Z \in \mathcal{Z}$.

In [74] any functional $\mathcal{D}: L^{2}(\Omega, \mathcal{F}, P) \rightarrow[0, \infty]$ with properties i), ii), iv), v) is called a deviation measure. Note however that these axioms of a deviation measure do not capture the monotonicity of the initial risk measure $\rho$. More precisely, the functional $\rho: L^{2}(\Omega, \mathcal{F}, P) \rightarrow \mathbb{R} \cup\{\infty\}$, defined by

$$
\rho(X):=E_{P}[-X]+\mathcal{D}(X)
$$

for a given deviation measure $\mathcal{D}$, will in general not be monotone as required in the definition of a monetary risk measure. Nevertheless, the dual characterization (56) holds for any lowersemicontinuous deviation measure $\mathcal{D}$ on $L^{2}(\Omega, \mathcal{F}, P)$; cf. [74], Theorem 1 .

For a related discussion of deviation-type risk functionals we refer to Pflug \& Römisch [73].

\section{Beyond law-invariance}

So far we have fixed a reference probability measure $P$ which is assumed to be known. Let us now consider a situation of model uncertainty where $P$ is replaced by a whole class $\mathcal{P}$ of probability measures on $(\Omega, \mathcal{F})$. In the face of such model ambiguity, it is plausible to focus on risk measures of the form

$$
\rho_{\mathcal{P}}(X):=\sup _{P \in \mathcal{P}} \rho_{P}(X)
$$

on $\mathcal{X}=L^{\infty}(\Omega, \mathcal{F})$, where each $\rho_{P}$ is a monetary risk measure on $\mathcal{X}$ which is law-invariant with respect to $P \in \mathcal{P}$. Clearly, $\rho_{\mathcal{P}}$ is again a monetary risk measure on $\mathcal{X}$, but in general it is no longer law-invariant. Such a robustification of risk measures or risk functionals is also discussed in Pflug, Pichler \& Wozabal [72], where $\mathcal{P}$ is specified as the class of all probability measures whose Wasserstein distance to a given reference measure $P$ does not exceed a certain threshold.

Example 6.1. Consider the robust extension of Value at Risk at level $\lambda$ defined by

$$
\operatorname{VaR}_{\mathcal{P}, \lambda}(X):=\sup _{P \in \mathcal{P}} \operatorname{VaR}_{P, \lambda}(X) .
$$

In this case, we have

$$
\operatorname{VaR}_{\mathcal{P}, \lambda}(X)=\inf \left\{m \in \mathbb{R} \mid \sup _{P \in \mathcal{P}} P[X+m<0] \leq \lambda\right\},
$$

i. e., $\mathrm{VaR}_{\mathcal{P}, \lambda}$ is computed in terms of the worst-case probability of a loss with respect to the class $\mathcal{P}$. Of course, there are other ways of aggregating Value at Risk for different models $P \in \mathcal{P}$, for example by taking a weighted sum or by combining weighted sums and suprema; $c f$. the discussion of Stressed Value at Risk and the resulting capital requirement in [2], pp. 14-15. 
Suppose now that each $\rho_{P}$ is a convex risk measure and admits a robust representation (12) with some penalty function $\alpha_{P}$. Then $\rho_{\mathcal{P}}$ will be a convex risk measure with robust representation

$$
\begin{aligned}
\rho_{\mathcal{P}}(X) & =\sup _{P \in \mathcal{P}} \sup _{Q \in \mathcal{M}_{1}}\left\{E_{Q}[-X]-\alpha_{P}(Q)\right\} \\
& =\sup _{Q \in \mathcal{Q}_{\mathcal{P}}}\left\{E_{Q}[-X]-\alpha_{\mathcal{P}}(Q)\right\},
\end{aligned}
$$

where the penalty function $\alpha_{\mathcal{P}}$ is given by

$$
\alpha_{\mathcal{P}}(Q):=\inf _{P \in \mathcal{P}} \alpha_{P}(Q)
$$

for $Q \in \mathcal{M}_{1}$, and

$$
\mathcal{Q}_{\mathcal{P}}:=\left\{Q \in \mathcal{M}_{1} \mid \alpha_{\mathcal{P}}(Q)<\infty\right\}
$$

is contained in $\mathcal{M}_{1}(\mathcal{P}):=\left\{Q \in \mathcal{M}_{1} \mid Q \ll P\right.$ for some $\left.P \in \mathcal{P}\right\}$.

\subsection{Robustified entropic risk measures}

Let us now look at the robustified versions of the convex and coherent entropic risk measures introduced in Subsection 4.6 .

Assumption 6.1. We assume that all measures $P \in \mathcal{P}$ are equivalent to some reference measure $R$ on $(\Omega, \mathcal{F})$, and that the family of densities

$$
\Phi_{\mathcal{P}}:=\left\{\frac{d P}{d R} \mid P \in \mathcal{P}\right\}
$$

is convex and weakly compact in $L^{1}(R)$.

Denote by

$$
H(Q \mid \mathcal{P}):=\inf _{P \in \mathcal{P}} H(Q \mid P)
$$

the relative entropy of $Q$ with respect to the class $\mathcal{P}$. Assumption 6.1 implies that for each $Q$ with $H(Q \mid \mathcal{P})<\infty$ there exists a unique measure $P_{Q} \in \mathcal{P}$, called the reverse entropic projection of $Q$ on $\mathcal{P}$, such that $H\left(Q \mid P_{Q}\right)=H(Q \mid \mathcal{P})$; cf. Csiszár \& Tusnády [22], and also Föllmer \& Gundel [37, Remark 2.10 and Proposition 2.14 for an extension to general $g$-divergences.

\subsubsection{The convex case}

The robust version $e_{\mathcal{P}, \gamma}$ of the convex entropic risk measure $e_{P, \gamma}$ is defined by

$$
e_{\mathcal{P}, \gamma}(X):=\sup _{P \in \mathcal{P}} e_{P, \gamma}(X)=\frac{1}{\gamma} \sup _{P \in \mathcal{P}} \log E_{P}\left[e^{-\gamma X}\right]
$$

for any $X \in \mathcal{X}$. Assumption 6.1 implies that the supremum is actually attained. Clearly, $e_{\mathcal{P}, \gamma}$ is again a convex risk measure, and its robust representation takes the form

$$
e_{\mathcal{P}, \gamma}(X)=\sup _{Q \in \mathcal{M}_{1}}\left\{E_{Q}[-X]-\frac{1}{\gamma} H(Q \mid \mathcal{P})\right\}
$$

Moreover, $e_{\mathcal{P}, \gamma}(X)$ is increasing in $\gamma$ with

$$
\lim _{\gamma \downarrow 0} e_{\mathcal{P}, \gamma}(X)=\max _{P \in \mathcal{P}} E_{P}[-X] \text { and } \lim _{\gamma \uparrow \infty} e_{\mathcal{P}, \gamma}(X)=\operatorname{ess} \sup _{R}(-X) .
$$




\subsubsection{The coherent case}

The robust extension $\rho_{\mathcal{P}, c}$ of the coherent entropic risk measure is defined by

$$
\rho_{\mathcal{P}, c}(X):=\sup _{Q \in \mathcal{M}_{1}: H(Q \mid \mathcal{P}) \leq c} E_{Q}[-X]
$$

for any $X \in \mathcal{X}$.

As shown in 38, Lemma 5.2 and Proposition 5.1, the supremum in 59 is attained, and we have

$$
\rho_{\mathcal{P}, c}(X)=\max _{P \in \mathcal{P}} \rho_{P, c}(X),
$$

in accordance with (57). More precisely:

Proposition 6.1. For

$$
c<-\log \max _{P \in \mathcal{P}} P[X=\operatorname{essinf} X]
$$

we have

$$
\begin{aligned}
\rho_{\mathcal{P}, c}(X) & =\max _{P \in \mathcal{P}} \min _{\gamma>0}\left\{\frac{c}{\gamma}+e_{P, \gamma}(X)\right\} \\
& =\frac{c}{\gamma_{c}}+e_{P_{c}, \gamma_{c}}(X) \\
& =E_{Q_{c}}[-X]
\end{aligned}
$$

where $Q_{c}$ denotes the measure in the exponential family of $P_{c}$ and $-X$ with parameter $\gamma_{c}$, and $\gamma_{c}>0$ is such that

$$
H\left(Q_{c} \mid \mathcal{P}\right)=H\left(Q_{c} \mid P_{c}\right)=c .
$$

If $c \geq-\log \max _{P \in \mathcal{P}} P[X=\operatorname{ess} \inf X]$, then

$$
\rho_{\mathcal{P}, c}(X)=\operatorname{ess} \sup (-X) .
$$

Note that Proposition 6.1 together with 58 implies

$$
\lim _{c \downarrow 0} \rho_{\mathcal{P}, c}(X)=\max _{P \in \mathcal{P}} E_{P}[-X] \quad \text { and } \quad \lim _{c \uparrow \infty} \rho_{\mathcal{P}, c}(X)=\operatorname{ess} \sup _{R}(-X) .
$$

Moreover, the estimates (34) and (21) yield

$$
\operatorname{VaR}_{\mathcal{P}, \lambda}(X) \leq \rho_{\mathcal{P},-\log \lambda}(X)
$$

\subsection{Asymptotics of robust entropic capital requirements}

Let us now focus on the asymptotic behavior of capital requirements for large portfolios in our present context of model ambiguity. Consider a portfolio of $n$ positions, described as random variables $X_{1}, \ldots, X_{n}$ in $\mathcal{X}=L^{\infty}(\Omega, \mathcal{F})$ which are independent and identically distributed under any $P \in \mathcal{P}$ and such that the variances $\sigma_{P}^{2}\left(X_{1}\right)$ of $X_{1}$ under $P$ satisfy $\inf _{P \in \mathcal{P}} \sigma_{P}\left(X_{1}\right)>0$.

For the robust version $e_{\mathcal{P}, \gamma}$ of the convex entropic risk measure our homogeneity assumption on the random variables $X_{1}, \ldots, X_{n}$ implies that the capital requirement for the aggregate position $S_{n}:=X_{1}+\ldots+X_{n}$ is given by

$$
e_{\mathcal{P}, \gamma}\left(S_{n}\right)=n e_{\mathcal{P}, \gamma}\left(X_{1}\right)
$$

Thus the capital requirements per position do not decrease as the portfolio becomes large, in analogy to Remark 4.1 .

Let us now look at the robust version $\rho_{\mathcal{P}, c}$ of the coherent entropic risk measures. In this case, the robustified capital requirements per position defined by

$$
\pi_{\mathcal{P}, n}:=\frac{1}{n} \rho_{\mathcal{P}, c}\left(S_{n}\right)
$$


converge to the robustified fair value

$$
\max _{P \in \mathcal{P}} E_{P}\left[-X_{1}\right],
$$

which appears as the risk-neutral limit both in $(58)$ and $(60)$. More precisely, we have

$$
\varlimsup_{n \uparrow \infty} \sqrt{n}\left(\pi_{\mathcal{P}, n}-\max _{P \in \mathcal{P}} E_{P}\left[-X_{1}\right]\right) \leq \sup _{P \in \mathcal{P}} \sigma_{P}\left(X_{1}\right) \sqrt{2 c} ;
$$

cf. 38, Proposition 4.3. This convergence result for robustified coherent entropic risk measures will be generalized in Theorem 7.3 .

\subsection{Robust loss probabilities and robust large deviations}

For a fixed capital requirement per contract $\pi$ such that

$$
\max _{P \in \mathcal{P}} E_{P}\left[-X_{1}\right]<\pi<\operatorname{ess} \sup \left(-X_{1}\right),
$$

we look at the robust loss probabilities

$$
\sup _{P \in \mathcal{P}} P\left[-\frac{1}{n} S_{n} \geq \pi\right]
$$

and their asymptotic behavior as the portfolio becomes large.

In a first step, we fix $n \in \mathbb{N}$ and take $c>0$ such that $\rho_{\mathcal{P}, c}\left(S_{n}\right) \leq n \pi$. Then inequality 61 implies $\operatorname{VaR}_{\mathcal{P}, \exp (-c)}\left(S_{n}\right) \leq n \pi$, i. e.,

$$
\sup _{P \in \mathcal{P}} P\left[S_{n}+n \pi<0\right] \leq \exp (-c) .
$$

In order to minimize this upper bound for the robust loss probability, we now determine the maximal tolerance level

$$
c_{\pi, n}:=\max \left\{c>0 \mid \frac{1}{n} \rho_{\mathcal{P}, c}\left(S_{n}\right) \leq \pi\right\} ;
$$

cf. [38, Proposition 5.2

Proposition 6.2. For a fixed premium $\pi$ the tolerance level $c_{\pi, n}$ is given by

$$
c_{\pi, n}=n I_{\mathcal{P}}(\pi)=n \Lambda_{\mathcal{P}}^{*}(\pi),
$$

where

$$
I_{\mathcal{P}}(\pi):=\min _{Q: E_{\mathcal{Q}}\left[-X_{1}\right]=\pi} H(Q \mid \mathcal{P})
$$

and

$$
\Lambda_{\mathcal{P}}^{*}(\pi)=\sup _{\gamma>0}\left\{\gamma \pi-\sup _{P \in \mathcal{P}} \log E_{P}\left[e^{-\gamma X_{1}}\right]\right\} .
$$

In particular, $I_{\mathcal{P}}$ coincides with the convex conjugate $\Lambda_{\mathcal{P}}^{*}$ of the convex function $\Lambda_{\mathcal{P}}$ defined by

$$
\Lambda_{\mathcal{P}}(\gamma):=\sup _{P \in \mathcal{P}} \log E_{P}\left[e^{-\gamma X_{1}}\right], \quad \gamma>0 .
$$

The preceding description of the maximal tolerance level $c_{\pi, n}$ yields a robust extension of the classical Cramér bounds for large deviations:

Corollary 6.1. For any $n \in \mathbb{N}$ we have

$$
\sup _{P \in \mathcal{P}} P\left[-\frac{1}{n} S_{n}>\pi\right] \leq \exp \left(-n I_{\mathcal{P}}(\pi)\right) .
$$

Moreover,

$$
\lim _{n \uparrow \infty} \frac{1}{n} \log \left(\sup _{P \in \mathcal{P}} P\left[-\frac{1}{n} S_{n}>\pi\right]\right)=-I_{\mathcal{P}}(\pi) .
$$


The inequality (63) follows immediately from 62 and the description of $c_{\pi, n}$ in Proposition 6.2 . On the other hand, Cramér's theorem yields

$$
\varliminf_{n \uparrow \infty} \frac{1}{n} \log P\left[-\frac{1}{n} S_{n}>\pi\right] \geq-\min _{Q: E_{Q}\left[-X_{1}\right]=\pi} H(Q \mid P)
$$

for any $P \in \mathcal{P}$. Combined with a minimax argument, this implies the robust lower bound

$$
\begin{aligned}
\frac{\lim }{n \uparrow \infty} \frac{1}{n} \log \left(\sup _{P \in \mathcal{P}} P\left[-\frac{1}{n} S_{n}>\pi\right]\right) & \geq \sup _{P \in \mathcal{P}}\left(-\min _{Q: E_{Q}\left[-X_{1}\right]=\pi} H(Q \mid P)\right) \\
& =-\min _{Q: E_{Q}\left[-X_{1}\right]=\pi} H(Q \mid \mathcal{P}) \\
& =-I_{\mathcal{P}}(\pi) ;
\end{aligned}
$$

cf., e.g., Terkelsen [79, Corollary 2. Together with the robust upper bound (63) this yields (64), i. e., the robust loss probability decreases to zero exponentially fast at the rate $I_{\mathcal{P}}(\pi)$. For related results on robust large deviations we refer to Sadowsky [76], Pandit \& Meyn [70], and Hu [57].

\section{Asymptotics for large portfolios}

Let us consider a portfolio of $n$ positions $X_{1}, \ldots, X_{n}$. Given a convex risk measure $\rho$, the capital which is required in order to make the aggregate position $S_{n}=X_{1}+\cdots+X_{n}$ acceptable is specified as $\rho\left(S_{n}\right)$, and

$$
\pi_{n}:=\frac{1}{n} \rho\left(S_{n}\right)
$$

is the resulting capital requirement per position. From an actuarial point of view, $\rho\left(S_{n}\right)$ can be seen as the aggregate premium which is needed to secure a portfolio of $n$ insurance contracts with losses $-X_{1}, \ldots,-X_{n}$, and then $\pi_{n}$ is the premium per contract.

Our aim is to show under which conditions the capital requirements per position decrease as the portfolio becomes large. We have seen that this does not happen if capital requirements are specified by a convex entropic risk measure $e_{\gamma}$; cf. Remark 4.1. On the other hand, it does happen if we use instead a coherent entropic risk measure $\rho_{c}$ or a truncated entropic risk measure $e_{\gamma, c}$; cf. Remark 4.2 and 4.3 In this section we look at this question from a more general point of view. First we consider the law-invariant case, and then we discuss an extension beyond law-invariance which takes model uncertainty into account.

\subsection{The law-invariant case}

Let $\rho$ be a normalized convex risk measure that is law-invariant with respect to a given probability measure $P$ on $(\Omega, \mathcal{F})$. As shown at the beginning of Section $5, \rho$ can then be regarded as a convex risk measure on $L^{1}(\Omega, \mathcal{F}, P)$, and it admits a representation

$$
\rho(X)=\sup _{\mu \in \mathcal{M}_{1}((0,1])}\left\{\int_{(0,1]} \operatorname{AVaR}_{\lambda}(X) \mu(d \lambda)-\beta(\mu)\right\}
$$

in terms of mixtures of Average Value at Risk; cf. Theorem 5.1

Assumption 7.1. We assume that the random variables $X_{1}, \ldots, X_{n}$ are independent and identically distributed under $P$, and that $X_{1}$ has exponential moments of any order, $i . e$.

$$
E_{P}\left[e^{\alpha\left|X_{1}\right|}\right]<\infty \text { for any } \alpha>0
$$

We also assume that the distribution of $X_{1}$ under $P$ is non-degenerate and denote by $\sigma_{P}^{2}\left(X_{1}\right)>0$ the variance of $X_{1}$ with respect to $P$. 
The following discussion can be extended beyond the i. i. d. case under much weaker conditions of homogeneity and weak dependence for the underlying sequence $X_{1}, \ldots, X_{n}$, as long as the standardized sums satisfy the central limit theorem and we retain control over their exponential moments.

Condition 66 ensures that the aggregate positions $S_{n}$ belong to the Orlicz heart $\mathcal{O}^{h}$ in 18 with respect to the Young function $h(x)=e^{x}-1$. In particular, $S_{n}$ admits a finite Orlicz norm $\|\cdot\|_{h}$, as defined in (14).

Since the positions are non-constant, law-invariance of the convex risk measure $\rho$ implies $\rho\left(S_{n}\right)>E_{P}\left[-S_{n}\right]$ as in $(55)$, and hence

$$
\pi_{n}>E_{P}\left[-X_{1}\right]
$$

i. e., the capital requirement per position is larger than the "fair premium". Our aim is to clarify the conditions that guarantee that the risk premia $\pi_{n}-E_{P}\left[-X_{1}\right]$ converge to 0 as $n$ becomes large, and to determine the rate of convergence.

\subsubsection{The coherent case}

Let us first focus on the coherent risk measures

$$
\rho_{\mathcal{M}}(X)=\sup _{\mu \in \mathcal{M}} \int_{0}^{1} \operatorname{AVaR}_{\lambda}(X) \mu(d \lambda), \quad \mathcal{M} \subseteq \mathcal{M}_{1}([0,1]),
$$

and on the associated capital requirements per position, or insurance premia per contract, given by

$$
\pi_{n}=\frac{1}{n} \rho_{\mathcal{M}}\left(S_{n}\right)
$$

For $\mathcal{M}=\{\mu\}$ we obtain the comonotonic risk measures

$$
\rho_{\mu}(X)=\int_{0}^{1} \operatorname{AVaR}_{\lambda}(X) \mu(d \lambda),
$$

and this reduces to Average Value at Risk at level $\lambda$ if we take $\mu=\delta_{\lambda}$. Note now that

$$
\operatorname{AVaR}_{0}\left(S_{n}\right)=\operatorname{ess} \sup \left(-S_{n}\right)=n \operatorname{ess} \sup \left(-X_{1}\right),
$$

hence

$$
\pi_{\delta_{0}, n}=\operatorname{ess} \sup \left(-X_{1}\right), \quad n \in \mathbb{N}
$$

and so the pooling of positions does not reduce the capital requirement per position. The same observation holds in the comonotonic case if $\mu[\{0\}]>0$. Indeed,

$$
\rho_{\mu}\left(S_{n}\right) \geq \mu[\{0\}] n \operatorname{ess} \sup \left(-X_{1}\right)+(1-\mu[\{0\}]) n E_{P}\left[-X_{1}\right]
$$

and this implies

$$
\underline{\lim _{n \uparrow \infty}}\left(\pi_{\mu, n}-E_{P}\left[-X_{1}\right]\right) \geq \mu[\{0\}]\left(\operatorname{ess} \sup \left(-X_{1}\right)-E_{P}\left[-X_{1}\right]\right)>0
$$

i. e., the desired convergence of $\pi_{\mu, n}$ to the "fair premium" $E_{P}\left[-X_{1}\right]$ does not take place.

We will thus limit the discussion to a class $\mathcal{M}$ of measure $\mu$ that are concentrated on $(0,1]$. Moreover, we require that $\mathcal{M}$ does not pile up to much mass near 0 , and this will be captured by the integrability condition

$$
\sup _{\mu \in \mathcal{M}} \int_{0}^{1} \log \left(\frac{1}{\lambda}\right) \mu(d \lambda)<\infty
$$


Remark 7.1. For the comonotonic risk measure $\rho_{\mu}$ in $(68)$ the integrability condition (69) reduces to

$$
\int_{0}^{1} \log \left(\frac{1}{\lambda}\right) \mu(d \lambda)<\infty .
$$

Recall that $\rho_{\mu}$ can be represented as the Choquet integral of the loss $-X$ with respect to the concave distortion $c=\psi \circ P$, where $\psi$ is defined by (45); cf. Subsection 5.1. Condition (70) holds if and only if the distortion function $\psi$ satisfies

$$
\int_{0}^{1} \log \left(\frac{1}{t}\right) \psi_{+}^{\prime}(t) d t<\infty
$$

cf. [39], Lemma 4.1. This is indeed the case for the concave distortions $\left\{\psi_{\lambda} \mid \lambda \geq 0\right\}$ proposed by Wang [82], where $\psi_{\lambda}$ is defined by $\psi_{\lambda}(0)=0$ and

$$
\psi_{\lambda}^{\prime}(t):=\frac{\varphi\left(\Phi^{-1}(t)+\lambda\right)}{\varphi\left(\Phi^{-1}(t)\right)}
$$

cf. [39], Proposition 4.1.

Using Young's inequality 15 with $h$ as above and its conjugate function $h^{*}$, one obtains the estimate

$$
\begin{aligned}
\left|\rho_{\mathcal{M}}(X)\right| & \leq 2\|X\|_{h} \sup _{\mu \in \mathcal{M}} \int_{(0,1]} \frac{1}{\lambda\left(h^{*}\right)^{-1}\left(\frac{1}{\lambda}\right)} \mu(d \lambda) \\
& \leq 2\|X\|_{h} \sup _{\mu \in \mathcal{M}} \int_{(0,1]} \log \left(\frac{1}{\lambda}\right) \mu(d \lambda) ;
\end{aligned}
$$

cf. 39], Lemma 3.1 and Remark 3.4. Thus condition 69 ensures finiteness of $\rho_{\mathcal{M}}$ on the Orlicz space $L^{h}(P) \supseteq \mathcal{O}^{h}$, and so the capital requirements $\rho_{\mathcal{M}}\left(S_{n}\right)$ and the corresponding premia $\pi_{n}$ are well defined and finite, due to our Assumption 7.1.

Theorem 7.1. Under condition $\sqrt{69}$ the premia $\pi_{n}$ converge to the fair premium $E_{P}\left[-X_{1}\right]$, and they do so at the rate $n^{-1 / 2}$. More precisely,

$$
\begin{aligned}
\lim _{n \uparrow \infty} \sqrt{n}\left(\pi_{n}-E_{P}\left[-X_{1}\right]\right) & =\sigma_{P}\left(X_{1}\right) \sup _{\mu \in \mathcal{M}} \int_{(0,1]} \operatorname{AVaR}_{\lambda}(Z) \mu(d \lambda) \\
& =\sigma_{P}\left(X_{1}\right) \sup _{\mu \in \mathcal{M}} \int_{(0,1]} \frac{1}{\lambda} \varphi\left(\Phi^{-1}(\lambda)\right) \mu(d \lambda),
\end{aligned}
$$

where $Z$ is standard normally distributed, and where $\varphi$ and $\Phi$ denote the density and the distribution function of the standard normal distribution.

Let us sketch the main arguments; for details we refer to [39. Consider first the building blocks $\mathrm{AVaR}_{\lambda}$ for $\lambda \in(0,1)$. Using cash invariance and positive homogeneity, we can write

$$
\begin{aligned}
\sqrt{n}\left(\pi_{\delta_{\lambda}, n}-E_{P}\left[-X_{1}\right]\right) & =\frac{1}{\sqrt{n}}\left(\operatorname{AVaR}_{\lambda}\left(S_{n}\right)-n E_{P}\left[-X_{1}\right]\right) \\
& =\sigma_{P}\left(X_{1}\right) \operatorname{AVaR}_{\lambda}\left(S_{n}^{*}\right)
\end{aligned}
$$

in terms of the standardized random variables

$$
S_{n}^{*}:=\frac{S_{n}-n E_{P}\left[X_{1}\right]}{\sqrt{n} \sigma_{P}\left(X_{1}\right)} .
$$

The central limit theorem yields weak convergence of the distributions of $S_{n}^{*}$ to the standard normal distribution. For any choice of the quantile functions $q_{n}$ of $S_{n}^{*}$, this implies pointwise convergence 
$\lim _{n \uparrow \infty} q_{n}(\alpha)=\Phi^{-1}(\alpha), \alpha \in(0,1)$, to the quantile function $\Phi^{-1}$ of a standard normally distributed random variable $Z$. Since $\int_{0}^{1}\left(q_{n}(\alpha)\right)^{2} d \alpha \equiv 1$, the sequence $\left(q_{n}\right)_{n \in \mathbb{N}}$ is uniformly integrable on the unit interval. Thus we get

$$
\lim _{n \uparrow \infty} \operatorname{AVaR}_{\lambda}\left(S_{n}^{*}\right)=-\frac{1}{\lambda} \lim _{n \uparrow \infty} \int_{0}^{\lambda} q_{n}(\alpha) d \alpha=-\frac{1}{\lambda} \int_{0}^{\lambda} \Phi^{-1}(\alpha) d \alpha=\frac{1}{\lambda} \varphi\left(\Phi^{-1}(\lambda)\right)=\operatorname{AVaR}_{\lambda}(Z),
$$

and so (71) holds for the class $\mathcal{M}=\left\{\delta_{\lambda}\right\}$ corresponding to $\mathrm{AVaR}_{\lambda}$.

For a general coherent risk measure $\rho_{\mathcal{M}}$, its representation (67) together with $(72)$ yields

$$
\sqrt{n}\left(\pi_{\mathcal{M}, n}-E_{P}\left[-X_{1}\right]\right)=\sigma_{P}\left(X_{1}\right) \sup _{\mu \in \mathcal{M}} \int_{(0,1]} \operatorname{AVaR}_{\lambda}\left(S_{n}^{*}\right) \mu(d \lambda) .
$$

It is thus enough to show that

$$
\lim _{n \uparrow \infty} \sup _{\mu \in \mathcal{M}} \int_{(0,1]} \operatorname{AVaR}_{\lambda}\left(S_{n}^{*}\right) \mu(d \lambda)=\sup _{\mu \in \mathcal{M}} \int_{(0,1]} \operatorname{AVaR}_{\lambda}(Z) \mu(d \lambda) .
$$

This follows by a careful application of Young's inequality, using Assumption 7.1 and the integrability condition 69 .

Example 7.1. Consider the coherent entropic risk measure $\rho_{c}$ at level $c>0$ defined by (31), and recall that its representing class $\mathcal{M}_{c}$ is given by [41]. By Corollary 3.1 in [39], the integrability condition (69) is satisfied for any class $\mathcal{M}$ such that

$$
\sup _{\mu \in \mathcal{M}} \int_{0}^{1} q_{\mu}(t) \log q_{\mu}(t) d t<\infty,
$$

where $q_{\mu}$ is defined in (42). In particular it holds for the class $\mathcal{M}_{c}$, and so the asymptotic behavior of the premia $\pi_{n}=\frac{1}{n} \rho_{c}\left(S_{n}\right)$ is described by (71). This is consistent with (35) since

$$
\sup _{\mu \in \mathcal{M}_{c}} \int_{0}^{1} \frac{1}{\lambda} \varphi\left(\Phi^{-1}(\lambda)\right) \mu(d \lambda)=\sqrt{2 c}
$$

cf. [39], Proposition 4.3.

\subsubsection{The convex case}

Let us now look at a general law-invariant convex risk measure $\rho$. Its representation $(65)$ takes the form

$$
\rho(X)=\sup _{\mu \in \mathcal{M}_{\rho}}\left\{\int_{(0,1]} \operatorname{AVaR}_{\lambda}(X) \mu(d \lambda)-\beta(\mu)\right\},
$$

where

$$
\mathcal{M}_{\rho}:=\left\{\mu \in \mathcal{M}_{1}((0,1]) \mid \beta(\mu)<\infty\right\} .
$$

Under the condition

$$
\sup _{\mu \in \mathcal{M}_{\rho}} \int_{(0,1]} \log \left(\frac{1}{\lambda}\right) \mu(d \lambda)<\infty,
$$

the coherent risk measure $\rho_{\mathcal{M}}$ has a natural extension from $L^{\infty}(P)$ to the Orlicz space $L^{h}(P)$, and the same is true for the convex risk measure $\rho \leq \rho_{\mathcal{M}_{\rho}}$.

In addition we are going to assume the condition

$$
\sup _{\mu \in \mathcal{M}_{\rho}} \beta(\mu)<\infty .
$$


This condition is clearly satisfied in the coherent case, since then we have $\beta \equiv 0$ on $\mathcal{M}_{\rho}$. In the convex case it holds if, in addition to 74 , the losses $X^{-}$of acceptable positions are bounded in the Orlicz norm $\|\cdot\|_{h}$, i. e.,

$$
\sup _{X \in \mathcal{A}_{\rho}}\left\|X^{-}\right\|_{h}<\infty
$$

cf. [39], Lemma 3.2. The following result says that the asymptotic behavior of the risk premia under the convex risk measure $\rho$ coincides with their behavior under the associated coherent risk measure $\rho_{\mathcal{M}}$ as described by Theorem 7.1, for the proof we refer to 39, Theorem 3.3.

Theorem 7.2. Consider a convex risk measure $\rho$ of the form (65) which satisfies conditions (74) and $(75)$. Then the premia $\pi_{n}:=\frac{1}{n} \rho\left(S_{n}\right)$ converge to the fair premium $E_{P}\left[-X_{1}\right]$, and

$$
\lim _{n \uparrow \infty} \sqrt{n}\left(\pi_{n}-E_{P}\left[-X_{1}\right]\right)=\sigma_{P}\left(X_{1}\right) \sup _{\mu \in \mathcal{M}_{\rho}} \int_{(0,1]} \frac{1}{\lambda} \varphi\left(\Phi^{-1}(\lambda)\right) \mu(d \lambda) .
$$

Example 7.2. Consider the truncated convex entropic risk measure $e_{\gamma, c}$ defined in (36). The assumptions of Theorem 7.2 are clearly satisfied. The associated coherent risk measure coincides with the coherent entropic risk measure $\rho_{c}$, and so the premia $\pi_{n}=\frac{1}{n} e_{\gamma, c}\left(S_{n}\right)$ behave as in Example 7.1. In view of (73) this is consistent with Remark 4.3.

\subsection{Beyond law-invariance}

Let us return to the situation of model ambiguity as described in Subsection 6, where the reference measure $P$ is replaced by a whole class $\mathcal{P}$ of probability measures on $(\Omega, \mathcal{F})$ that satisfies Assumption 6.1.

For a given measure $P \in \mathcal{P}$ and a subset $\mathcal{M} \subseteq \mathcal{M}_{1}((0,1])$, we denote by $\rho_{P, \mathcal{M}}$ the coherent risk measure given by

$$
\rho_{P, \mathcal{M}}(X)=\sup _{\mu \in \mathcal{M}} \int_{(0,1]} \operatorname{AVaR}_{P, \lambda}(X) \mu(d \lambda) .
$$

The robustified version is defined as

$$
\rho_{\mathcal{P}, \mathcal{M}}(X):=\sup _{P \in \mathcal{P}} \rho_{P, \mathcal{M}}(X)=\sup _{P \in \mathcal{P}} \sup _{\mu \in \mathcal{M}} \int_{(0,1]} \operatorname{AVaR}_{P, \lambda}(X) \mu(d \lambda),
$$

and we denote by

$$
\pi_{\mathcal{P}, n}:=\frac{1}{n} \rho_{\mathcal{P}, \mathcal{M}}\left(S_{n}\right) .
$$

the corresponding robustified premia.

Let us now state a convergence result which extends both Theorem 7.1 and Subsection 6.2.

Assumption 7.2. The random variables $X_{1}, \ldots, X_{n}$ are $i . i$. $d$. under any $P \in \mathcal{P}$, and the exponential moments of $X_{1}$ are bounded uniformly in $P \in \mathcal{P}$, i. e.,

$$
\sup _{P \in \mathcal{P}} E_{P}\left[e^{\alpha\left|X_{1}\right|}\right]<\infty \quad \text { for any } \alpha>0 .
$$

Moreover, the variances $\sigma_{P}^{2}\left(X_{1}\right)$ of $X_{1}$ under $P$ satisfy $\inf _{P \in \mathcal{P}} \sigma_{P}\left(X_{1}\right)>0$.

For the class of mixing measures $\mathcal{M}$ we impose again the integrability condition (69). Under these conditions, the robustified premia $\pi_{\mathcal{P}, n}$ converge to the robust fair premium as the portfolio becomes large, and they do so at the rate $n^{-1 / 2}$; cf. [39], Theorem 5.1. More precisely:

Theorem 7.3. The robustified risk premia $\pi_{\mathcal{P}, n}-\sup _{P \in \mathcal{P}} E_{P}\left[-X_{1}\right]$ converge to 0 , and

$$
\varlimsup_{n \uparrow \infty} \sqrt{n}\left(\pi_{\mathcal{P}, n}-\sup _{P \in \mathcal{P}} E_{P}\left[-X_{1}\right]\right) \leq \sup _{P \in \mathcal{P}} \sigma_{P}\left(X_{1}\right) \sup _{\mu \in \mathcal{M}} \int_{(0,1]} \frac{1}{\lambda} \varphi\left(\Phi^{-1}(\lambda)\right) \mu(d \lambda) .
$$




\section{Risk measures and actuarial premium principles}

There are close connections between the literature on convex and coherent risk measures in mathematical finance and the actuarial literature on premium principles. In fact, convex risk measures already appear in 1985 on the actuarial side, up to a change of sign and in the form of a convex premium principle.

Let $\rho$ be a convex risk measure on $\mathcal{X}=L^{\infty}(\Omega, \mathcal{F})$. We assume that $\rho$ is finite and normalized to $\rho(0)=0$. Now consider the functional $H$ on $\mathcal{X}$ defined by

$$
H(X):=\rho(-X)
$$

Clearly, $H$ has the following properties:

i) $H(0)=0$,

ii) $H(X+m)=H(X)+m$ for $X \in \mathcal{X}$ and $m \in \mathbb{R}$,

iii) $X \leq Y \quad \Longrightarrow \quad H(X) \leq H(Y)$,

iv) $H$ is convex on $\mathcal{X}$.

Note also that $H$ is continuous from below if $\rho$ is continuous from above.

Such functionals $H$ were introduced by Deprez \& Gerber [28] in an actuarial context as "convex principles of premium calculation". More precisely, a functional $H: \mathcal{X} \rightarrow \mathbb{R}$ is called a convex principle in [28] if it satisfies conditions i) (normalization), ii) (translation property), and iv) (convexity). It is then shown that the additional condition iii) (monotonicity) is implied by the "no ripoff" condition

iii') $H(X) \leq \sup X$.

Indeed, if $X \leq Y$ then convexity together with i) and iii') implies

$$
H(\lambda X)=H\left(\lambda Y+(1-\lambda) \frac{\lambda}{1-\lambda}(X-Y)\right) \leq \lambda H(Y)
$$

for any $\lambda \in(0,1)$. In view of ii) we may assume $X \geq 0$. Letting $\lambda$ tend to 1 we get $H(X) \leq H(Y)$ if we also assume that $H$ is continuous from below.

The notion of a convex risk measure thus clearly appears in [28], up to a change of sign. This change of sign is due to the actuarial interpretation: Here $X$ describes the possible claims of an "insurance risk". The corresponding financial position of the insurer is then given by $-X$, and so the premium $H(X)$ is determined as the capital that is required to make that position acceptable.

If the initial risk measure $\rho$ is even coherent then the corresponding convex principle $H$ is proportional, i.e., $H(\lambda X)=\lambda H(X)$ for $\lambda \geq 0$. As observed in [28, this additional proportionality is equivalent to subadditivity of the convex principle $H$, i. e.,

$$
H(X+Y) \leq H(X)+H(Y)
$$

in accordance with the equivalence pointed out before Remark 2.1 .

From now on we fix a probability measure $P$ on $(\Omega, \mathcal{F})$ such that $(\Omega, \mathcal{F}, P)$ is atomless. In the law-invariant case the premium $H(X)$ only depends on the law of $X$ under $P$, and $H$ is then called a classical premium principle in [28]. In this classical case the connections between risk measures and actuarial premium principles are particularly strong; cf., e.g., Young [85], Kaas, Goovaerts, Dhaene \& Denuit 62], Goovaerts \& Laeven [53] and Goovaerts, Kaas, Dhaene \& Tang [51]. This is illustrated by the following examples. Note, however, that the robustification of law-invariant risk measures in Section 6 suggests a similar robustification of classical premium principles. 


\subsection{Entropic risk measures and the Esscher principle}

The premium principle

$$
H_{\gamma}(X):=\frac{1}{\gamma} \log E_{P}\left[e^{\gamma X}\right]
$$

which corresponds to the convex entropic risk measure $e_{\gamma}$ in $(28)$, is usually called the exponential principle. It can be characterized as the only convex premium principle which is at the same time a certainty equivalent with respect to some increasing continuous function $u$; this follows from de Finetti's criterion as explained in Subsection 4.6.1.

The exponential principle $H_{\gamma}$ satisfies the zero utility principle with respect to the exponential utility function $u_{\gamma}(x):=\frac{1}{\gamma}\left(1-e^{-\gamma x}\right)$, i. e.,

$$
E_{P}[u(H(X)-X)]=u(0)
$$

and it is additive on independent risks. Conversely, any premium principle which satisfies the zero utility principle and is additive for independent risks must be of the form $H_{\gamma}$ for some $\gamma \geq 0$, including the linear case $\gamma=0$; cf. Gerber [46].

Now consider the Esscher principle, defined by

$$
\begin{aligned}
H(X): & =E_{P}\left[X e^{\gamma X}\right]\left(E_{P}\left[e^{\gamma X}\right]\right)^{-1} \\
& =E_{Q_{X, \gamma}}[X]
\end{aligned}
$$

where $Q_{X, \gamma}$ denotes the measure with parameter $\gamma>0$ in the exponential family induced by $P$ and $X$. The Esscher principle is additive on independent risks. Conversely, as shown in [47, premium principles that are additive on independent risks and satisfy some additional monotonicity property can be characterized as mixtures of Esscher principles; see also [53] for a related characterization in terms of exponential principles.

The Esscher premium principle is neither convex nor monotone. Let us now compare it with the premium principle corresponding to the coherent entropic risk measure $\rho_{c}$ in Subsection 4.6.2. Here we have

$$
H(X)=\rho_{c}(-X)=E_{Q_{X, \gamma_{c}}}[X]
$$

where the parameter $\gamma_{c}$ is taken such that the corresponding measure in the exponential family has relative entropy $H\left(Q_{X, \gamma_{c}} \mid P\right)=c$. In contrast to the Esscher principle, the parameter $\gamma_{c}$ now depends both on $c$ and on the insurable risk $X$. But this modification has the effect that the resulting premium principle is monotone, convex, and proportional.

In this subsection we have restricted the discussion to the law-invariant case, and so the Esscher principle was only stated in its classical form $(77)$. But if a position $X$ is seen in a wider economic context then the exponential tilting in (77) may involve some other random variable $Z$ instead of $X$. In this case, the classical premium principle (77) is replaced by

$$
H(X)=E_{Q Z, \gamma}[X]
$$

where $Q_{Z, \gamma}$ belongs to the exponential family induced by $P$ and $Z$. Consider, for example, a reinsurance market with $n$ agents having initial risks $X_{1}, \ldots, X_{n}$ and exponential utility functions with parameters $\gamma_{1}, \ldots, \gamma_{n}$. As shown by Bühlmann [15, equilibrium prices in a Pareto-optimal risk exchange are given by an extended Esscher principle of the form $(78)$, where $Z:=X_{1}+\ldots+X_{n}$ and $\gamma=\left(\sum_{i=1}^{n} \gamma_{i}^{-1}\right)^{-1}$. For variants and extensions of the Esscher principle in the context of mathematical finance we refer to Gerber \& Shiu [48, 49], Bühlmann, Delbaen, Embrechts \& Shiryaev [16], Goovaerts \& Laeven [53], and Labuschagne \& Offwood 67]. 


\subsection{Haezendonck risk measures and the Orlicz premium principle}

Let $h$ be Young function as defined in Subsection 3.5 with $h(1)=1$. For $\lambda \in(0,1]$, the root $H_{\lambda}^{O}(X):=a$ of the equation

$$
E_{P}\left[h\left(\frac{X}{a}\right)\right]=\lambda
$$

is called the Orlicz premium of the risk $X$ at level $\lambda$. Using the convention $H_{\lambda}^{O}(0)=0$, the Orlicz premium is uniquely defined and finite for all $X \in L_{+}^{\infty}(\Omega, \mathcal{F}, P)$, or more generally for all $X \geq 0$ in the Orlicz space $L^{h}$ as soon as $h$ satisfies $\sup _{x>0} h(c x) / h(x)<\infty$ for some $c>0$. Note that the premium $H_{\lambda}^{O}$ coincides with the Orlicz norm $\|\cdot\|_{h_{\lambda}}$ taken with respect to the Young function $h_{\lambda}(x)=h(x) / \lambda$.

For $\lambda=1$ the Orlicz premium principle was proposed by Haezendonck \& Goovaerts [54] as a multiplicative version of a certainty equivalent. More precisely, if $h$ is viewed as a loss function and if the insurer is indifferent between the normalized loss $X / a$ and the benchmark 1 in the sense that

$$
E_{P}\left[h\left(\frac{X}{a}\right)\right]=h(1)=1,
$$

then the premium should be given by $H_{1}^{O}(X):=a$. For $\lambda<1$ the Orlicz premium $H_{\lambda}^{O}(X)$ can be interpreted in the same manner, taking into account an additional safety loading as specified by $\lambda$; cf. 51 .

An Orlicz premium principle satisfies properties i), iii) and iv) but in general not the translation property ii), and so it does not arise from a monetary risk measure $\rho$ as in $(76)$. On the other hand, it yields a simple bound for the loss probability, since

$$
P\left[X>H_{\lambda}^{O}(X)\right] \leq E_{P}\left[h\left(\frac{X}{H_{\lambda}^{O}(X)}\right)\right]=\lambda
$$

We now describe a modification of the Orlicz premium principle which does correspond to a convex risk measure, namely to the Haezendonck risk measure in Subsection 4.5. Let $H_{\lambda}(X, x):=a$ denote the solution of

$$
E_{P}\left[h\left(\frac{(X-x)^{+}}{a-x}\right)\right]=\lambda
$$

if $x<\operatorname{ess} \sup X$, and define $H_{\lambda}(X, x):=\infty$ otherwise. In this case, the loss probability resulting from charging the premium $H_{\lambda}(X, x)$ is given by

$$
P\left[X>H_{\lambda}(X, x)\right]=P\left[X-x>H_{\lambda}(X, x)-x\right] \leq E_{P}\left[h\left(\frac{(X-x)^{+}}{H_{\lambda}(X, x)-x}\right)\right]=\lambda .
$$

Let us now consider the minimal premium

$$
H_{\lambda}(X):=\inf _{x \in \mathbb{R}} H_{\lambda}(X, x)
$$

The resulting premium principle $H_{\lambda}$ satisfies properties i)-iv), and it takes the form $H_{\lambda}(X)=$ $\rho_{\lambda}(-X)$ for the coherent Haezendonck risk measure $\rho_{\lambda}$ corresponding to the Young function $h_{\lambda}(x)=h(x) / \lambda$; cf. [51] and Bellini \& Rosazza Gianin [9, 10]. As shown in [9], the Haezendonck risk measure $\rho_{\lambda}$ admits the alternative representation

$$
\rho_{\lambda}(X)=\inf _{x \in \mathbb{R}}\left\{H_{\lambda}^{O}\left((x-X)^{+}\right)-x\right\}=\inf _{x \in \mathbb{R}}\left\{\left\|(x-X)^{+}\right\|_{h_{\lambda}}-x\right\}
$$

in terms of Orlicz premia and Orlicz norms, and this translates into an analogous representation of the premium principle $H_{\lambda}$. 


\subsection{Comonotonic risk measures and Wang's premium principle}

Consider the premium principle $H$ corresponding via $H(X):=\rho(-X)$ to a normalized monetary risk measure $\rho$ that is positively homogeneous and law-invariant.

If $\rho$ is also convex and hence coherent, then $\rho$ respects second order stochastic dominance as stated in (51). In actuarial terms, this translates into the condition that the convex premium principle $H$ preserves the stop-loss order:

$$
E_{P}\left[(X-c)^{+}\right] \leq E_{P}\left[(Y-c)^{+}\right] \text {for all } c \in \mathbb{R} \quad \Longrightarrow \quad H(X) \leq H(Y) .
$$

Indeed, in view of 48 the left-hand side is equivalent to $-X \succeq_{(2)}-Y$, and so it implies $\rho(-X) \leq$ $\rho(-Y)$, due to 50$)$. Conversely, if $H$ preserves the stop-loss order then it is enough to require comonotonic subadditivity of $H$ as in 46 in order to guarantee that $H$ is a convex premium principle; cf. Subsection 5.2 .

If comonotonic subadditivity is strenghtened to comonotonic additivity of $H$ as in (53) then the underlying coherent risk measure $\rho$ is comonotonic, and we are in the situation of Subsection 5.1. In particular, $\rho$ takes the form 444. Thus $H$ is given by the Choquet integral

$$
H(X)=\int X d c
$$

with respect to some concave distortion $c=\psi \circ P$ of the underlying probability measure $P$. In the actuarial literature this is often called Wang's premium principle; cf. [82. Here we have derived it in the context of law-invariant coherent risk measures, starting with the Kusuoka representation (38. For a direct axiomatic characterization, with or without concavity of the distortion function $\psi$, and for the connection to Yaari's [84 dual theory of choice and to the earlier literature on Choquet integrals we refer to Panjer, Young \& Wang [71] and Denneberg [27].

The distortion function $\psi(x)=x^{p}$ for $p \in(0,1)$ yields the proportional hazards premium principle; cf., e.g., Wang [81. Another choice is Wang's distortion function $\psi(x)=\Phi\left(\Phi^{-1}(x)+\lambda\right)$, defined in terms of the cumulative distribution function of the standard normal distribution and a real parameter $\lambda$, cf. e.g., [82 and Remark 7.1. For $\psi(x)=1-(1-x)^{n}$ we obtain the actuarial form of the risk measure MINVAR in Example 5.2, namely

$$
H(X)=E_{P}\left[\max \left\{X_{1}, \ldots, X_{n}\right\}\right]
$$

where $X_{1}, \ldots, X_{n}$ are independent copies of $X$.

\section{Convex risk measures and robust preferences}

In this section we describe the connection between convex risk measures and the numerical representation of preferences in the face of model uncertainty.

Consider a preference order $\succeq$ on the space $\mathcal{X}$ of financial positions. Typically, $\succeq$ will admit a numerical representation, i. e.,

$$
X \succeq Y \quad \Longleftrightarrow \quad U(X) \geq U(Y)
$$

where $U$ is some real-valued utility functional on $\mathcal{X}$; cf. Alt 5 for a pioneering contribution and, e. g., [42, Chapter 2, and the references therein for later developments. In the paradigm of expected utility, the functional $U$ takes the form

$$
U(X)=E_{P}[u(X)]=\int u(x) \mu_{X}(d x)
$$

where $P$ is a probability measure on $(\Omega, \mathcal{F}), \mu_{X}$ denotes the distribution of $X$ under $P$, and $u$ is continuous and strictly increasing. Moreover, risk aversion is characterized by concavity of 
the function $u$. The axioms of "rationality" as formulated by von Neumann \& Morgenstern 80. characterize such preferences at the level of lotteries $\mu_{X}$, and a corresponding characterization at the level of positions $X$ was given by Savage [77.

Lotteries and positions can be seen as special cases of stochastic kernels $\tilde{X}$ from $(\Omega, \mathcal{F})$ to $\mathbb{R}$, often called acts or horse race lotteries; cf., e.g., Kreps 64. Indeed, a lottery $\mu$ corresponds to the kernel $\tilde{X}(\omega, \cdot) \equiv \mu$, a position $X$ to the kernel $\tilde{X}(\omega, \cdot)=\delta_{X(\omega)}$. Let us now fix the class $\tilde{\mathcal{X}}$ of all stochastic kernels $\tilde{X}(\omega, d x)$ for which there exists some constant $c$ such that $\tilde{X}(\omega,[-c, c])=1$ for all $\omega \in \Omega$. For preferences $\succeq$ on $\tilde{\mathcal{X}}$, Anscombe \& Aumann [6] have formulated a version of the rationality axioms which is equivalent to a numerical representation by an expected utility functional of the form

$$
\tilde{U}(\tilde{X})=E_{P}\left[\int u(x) \tilde{X}(\cdot, d x)\right] .
$$

Let now $\rho$ be a convex risk measure on $L^{\infty}(\Omega, \mathcal{F})$ with robust representation $(9)$, and take some increasing continuous function $u: \mathbb{R} \rightarrow \mathbb{R}$. Consider the utility functional $\tilde{U}: \tilde{\mathcal{X}} \rightarrow \mathbb{R}$ defined by

$$
\tilde{U}(\tilde{X}):=-\rho\left(\int u(x) \tilde{X}(\cdot, d x)\right)=\inf _{Q \in \mathcal{M}_{1}}\left\{E_{Q}\left[\int u(x) \tilde{X}(\cdot, d x)\right]+\alpha(Q)\right\}
$$

where the linear functional $E_{P}$ in 80 is replaced by the concave functional $-\rho$. The preference order $\succeq$ on $\tilde{\mathcal{X}}$ defined by

$$
\tilde{X} \succeq \tilde{Y} \quad: \Longleftrightarrow \tilde{U}(\tilde{X}) \geq \tilde{U}(\tilde{Y})
$$

satisfies the following properties:

- Monotonicity: The preference order $\succeq$ on $\tilde{\mathcal{X}}$ is monotone with respect to the embedding of the space of standard lotteries $\mathcal{M}_{1, c}(\overline{\mathbb{R}})$ in $\tilde{\mathcal{X}}$, i. e.,

$$
\tilde{X}(\omega, \cdot) \succeq_{(1)} \tilde{Y}(\omega, \cdot) \text { for all } \omega \in \Omega \quad \Longrightarrow \quad \tilde{X} \succeq \tilde{Y},
$$

where $\succeq_{(1)}$ denotes first order stochastic dominance; cf. Section 5.2 .

- Archimedian axiom: For $\tilde{X}, \tilde{Y}, \tilde{Z} \in \tilde{\mathcal{X}}$ with $\tilde{Z} \succeq \tilde{Y} \succeq \tilde{X}$ there exist $\alpha, \beta \in(0,1)$ such that

$$
\alpha \tilde{Z}+(1-\alpha) \tilde{X} \succeq \tilde{Y} \succeq \beta \tilde{Z}+(1-\beta) \tilde{X} .
$$

- Weak certainty independence: If for $\tilde{X}, \tilde{Y} \in \tilde{\mathcal{X}}$ and for some $\nu \in \mathcal{M}_{1, c}(\mathbb{R})$ and $\alpha \in(0,1]$ we have $\alpha \tilde{X}+(1-\alpha) \nu \succeq \alpha \tilde{Y}+(1-\alpha) \nu$, then

$$
\alpha \tilde{X}+(1-\alpha) \mu \succeq \alpha \tilde{Y}+(1-\alpha) \mu \text { for all } \mu \in \mathcal{M}_{1, c}(\mathbb{R}) .
$$

- Uncertainty aversion: If $\tilde{X}, \tilde{Y} \in \tilde{\mathcal{X}}$ are equivalent under $\succeq$, then

$$
\alpha \tilde{X}+(1-\alpha) \tilde{Y} \succeq \tilde{X} \quad \text { for all } \alpha \in[0,1] .
$$

In order to motivate the axiom of uncertainty aversion, let us look at the following simple example; cf., e.g., 42, Remark 2.76.

Example 9.1. Consider the two acts $\tilde{Z}_{0}, \tilde{Z}_{1}$ on $\Omega:=\{0,1\}$ defined by

$$
\tilde{Z}_{i}(\omega):=\delta_{1000} \cdot 1_{\{i\}}(\omega)+\delta_{0} \cdot 1_{\{i-1\}}(\omega), \quad i=0,1 .
$$

Both acts involve the same kind of Knightian uncertainty, and so it is natural to assume that they are equivalent with respect to the given preference order $\succeq$ on $\tilde{\mathcal{X}}$. In the case of uncertainty 
aversion, any convex combination $\tilde{Y}:=\alpha \tilde{Z}_{0}+(1-\alpha) \tilde{Z}_{1}$ is preferred over both $\tilde{Z}_{0}$ and $\tilde{Z}_{1}$. Here $\tilde{Y}$ takes the form

$$
\tilde{Y}(\omega)= \begin{cases}\alpha \delta_{1000}+(1-\alpha) \delta_{0} & \text { for } \omega=1 \\ \alpha \delta_{0}+(1-\alpha) \delta_{1000} & \text { for } \omega=0\end{cases}
$$

Thus model uncertainty is reduced in favor of risk, since the unknown probability of success is now known to be bounded by $\alpha$ and $1-\alpha$. For $\alpha=\frac{1}{2}$, the resulting lottery $\tilde{Y}(\omega)=\frac{1}{2}\left(\delta_{1000}+\delta_{0}\right)$ is even independent of the scenario $\omega$, i. e., Knightian uncertainty is completely replaced by the risk of a classical coin toss.

Conversely, Maccheroni, Marinacci \& Rustichini [68] have shown that the preceding four axioms imply that preferences can be represented by a utility functional $\tilde{U}$ of the form $(81)$, where $\rho$ is a convex risk measure on $L^{\infty}(\Omega, \mathcal{F})$; cf. also Föllmer, Schied \& Weber [43] and [42], Theorem 2.88. While risk aversion corresponds to concavity of the function $u$, the convexity of the risk measure $\rho$ captures a different behavioral feature, namely uncertainty aversion as illustrated in the preceding example.

Weak certainty independence can be strengthened to

- Full certainty independence: For all $\tilde{X}, \tilde{Y} \in \tilde{\mathcal{X}}, \mu \in \mathcal{M}_{1, c}(\mathbb{R})$, and $\alpha \in(0,1]$ we have

$$
\tilde{X} \succeq \tilde{Y} \quad \Longrightarrow \quad \alpha \tilde{X}+(1-\alpha) \mu \succeq \alpha \tilde{Y}+(1-\alpha) \mu .
$$

In this case the risk measure $\rho$ in 81 is actually coherent, and 81 reduces to the utility functional

$$
\tilde{U}(\tilde{X})=\inf _{Q \in \mathcal{Q}_{\rho}} E_{Q}\left[\int u(x) \tilde{X}(\cdot, d x)\right]
$$

cf. Gilboa \& Schmeidler [50] and, e.g., [42], Theorem 2.86. Under the additional assumptions of law-invariance and comonotonicity, the right-hand side can be described as a Choquet integral with respect to a concave distortion of an underlying probability measure $P$; cf. Section 8 and also Yaari's "dual theory of choice" 84.

Thus convex and coherent risk measures play a crucial role in recent advances in the theory of preferences in the face of risk and uncertainty. For further extensions, where the cash-invariance of $\rho$ is replaced by a weaker condition of cash-subadditivity, we refer to Cerreia-Vioglio, Maccheroni, Marinacci \& Montrucchio [18] and Drapeau \& Kupper [32].

\section{References}

[1] The Turner review - A regulatory response to the global banking crisis, published by the British Financial Services Authority, www.fsa.gov.uk/pubs/other/turner_review.pdf, 2009.

[2] Revisions to the Basel II market risk framework, published by the Basel Committee on Banking Supervision, www.bis.org/publ/bcbs158.pdf, 2009.

[3] B. Acciaio, H. Föllmer, and I. Penner. Risk assessment for uncertain cash flows: Model ambiguity, discounting ambiguity, and the role of bubbles. Finance Stoch., To appear 2012.

[4] B. Acciaio and I. Penner. Dynamic risk measures. In Advanced mathematical methods for finance, Eds. G. Di Nunno and B. Øksendal, pages 1-34. Springer, Heidelberg, 2011.

[5] F. Alt. Über die Messbarkeit des Nutzens. Zeitschrift für Nationalökonomie, 7(2):161-169, 1936. English Translation: On the measurability of utility. In: Preferences, Utility, and Demand (Ed. Chipman, Hurwicz, Richter, Sonnenschein), Harcourt Brace Jovanovich, New York, 1971.

[6] F. J. Anscombe and R. J. Aumann. A definition of subjective probability. Ann. Math. Statist., 34:199205, 1963.

[7] P. Artzner, F. Delbaen, J.-M. Eber, and D. Heath. Coherent measures of risk. Math. Finance, 9(3):203-228, 1999. 
[8] P. Artzner, F. Delbaen, and P. Koch-Medina. Risk measures and efficient use of capital. Astin Bull., 39(1):101-116, 2009.

[9] F. Bellini and E. Rosazza Gianin. On Haezendonck risk measures. Journal of Banking Es Finance, 32(6):986-994, 2008.

[10] F. Bellini and E. Rosazza Gianin. Optimal portfolios with Haezendonck risk measures. Statist. Decisions, 26(2):89-108, 2008.

[11] A. Ben-Tal and M. Teboulle. Penalty functions and duality in stochastic programming via $\phi$-divergence functionals. Math. Oper. Res., 12(2):224-240, 1987.

[12] A. Ben-Tal and M. Teboulle. An old-new concept of convex risk measures: the optimized certainty equivalent. Math. Finance, 17(3):449-476, 2007.

[13] S. Biagini and M. Frittelli. On the extension of the Namioka-Klee theorem and on the Fatou property for risk measures. In Optimality and risk-modern trends in mathematical finance, pages 1-28. Springer, Berlin, 2009.

[14] D. Brown, E. De Giorgi, and M. Sim. Dual representation of choice and aspirational preferences. Preprint, available under: http://papers.ssrn.com/sol3/papers.cfm?abstractid=1406399, 2010.

[15] H. Bühlmann. An economic premium principle. Astin Bull., 11(1):52-60, 1980/81.

[16] H. Bühlmann, F. Delbaen, P. Embrechts, and A. N. Shiryaev. No-arbitrage, change of measure and conditional Esscher transforms. CWI Quarterly, 9(4):291-317, 1996. Mathematics of Finance, Part I.

[17] C. Burgert and L. Rüschendorf. Consistent risk measures for portfolio vectors. Insurance Math. Econom., 38(2):289-297, 2006.

[18] S. Cerreia-Vioglio, F. Maccheroni, M. Marinacci, and L. Montrucchio. Risk measures: rationality and diversification. Math. Finance, 21(4):743-774, 2011.

[19] P. Cheridito and T. Li. Dual characterization of properties of risk measures on Orlicz hearts. Math. Financ. Econ., 2(1):29-55, 2008.

[20] P. Cheridito and T. Li. Risk measures on Orlicz hearts. Math. Finance, 19(2):189-214, 2009.

[21] A. Cherny and D. Madan. New measures for portfolio evaluation. Rev. Financ. Stud., 22(7):2571-2606, 2009.

[22] I. Csiszár and G. Tusnády. Information geometry and alternating minimization procedures. Statist. Decisions, suppl. 1:205-237, 1984. Recent results in estimation theory and related topics.

[23] R.-A. Dana. A representation result for concave Schur concave functions. Math. Finance, 15(4):613$634,2005$.

[24] B. De Finetti. Sul concetto di media. Giorn. Ist. Ital. Attuari, 2:369-396, 1931.

[25] F. Delbaen. Coherent risk measures. Cattedra Galileiana. Scuola Normale Superiore, Classe di Scienze, Pisa, 2000.

[26] F. Delbaen. Coherent risk measures on general probability spaces. In Advances in Finance and Stochastics, pages 1-37. Springer, Berlin, 2002.

[27] D. Denneberg. Non-additive measure and integral, volume 27 of Theory and Decision Library. Series B: Mathematical and Statistical Methods. Kluwer Academic Publishers Group, Dordrecht, 1994.

[28] O. Deprez and H. U. Gerber. On convex principles of premium calculation. Insurance Math. Econom., 4(3):179-189, 1985.

[29] J. Dhaene, A. Kukush, and M. Pupashenko. On the characterization of premium principle with respect to pointwise comonotonicity. Theory Stoch. Process., 12(3-4):26-42, 2006.

[30] J. Dhaene, S. Vanduffel, M. J. Goovaerts, R. Kaas, Q. Tang, and D. Vyncke. Risk measures and comonotonicity: a review. Stoch. Models, 22(4):573-606, 2006.

[31] S. Drapeau. Risk preferences and their robust representation. PhD thesis, Humboldt-Universität zu Berlin, available under: http://edoc.hu-berlin.de/dissertationen/drapeau-samuel-2010 -04-30/PDF/drapeau.pdf, 2010. 
[32] S. Drapeau and M. Kupper. Risk preferences and their robust representation. Preprint, available under: www .math.hu-berlin.de/ kupper/index.php?id=publications-and-preprint, 2010.

[33] S. Drapeau, M. Kupper, and R. Reda. A note on robust representations of law-invariant quasi-convex functions. Adv. Math. Econ., 15:27-39, 2011.

[34] J. Dunkel and S. Weber. Stochastic root finding and efficient estimation of convex risk measures. Oper. Res., 58(5):1505-1521, 2010.

[35] D. Filipović and G. Svindland. The canonical model space for law-invariant convex risk measures is $L^{1}$. To appear in Math. Finance.

[36] D. Filipović and N. Vogelpoth. A note on the Swiss solvency test risk measure. Insurance Math. Econom., 42(3):897-902, 2008.

[37] H. Föllmer and A. Gundel. Robust projections in the class of martingale measures. Illinois J. Math., 50(1-4):439-472 (electronic), 2006.

[38] H. Föllmer and T. Knispel. Entropic risk measures: coherence vs. convexity, model ambiguity, and robust large deviations. Stoch. Dyn., 11(2-3):333-351, 2011.

[39] H. Föllmer and T. Knispel. Convex capital requirements for large portfolios. In Stochastic Analysis and its Applications to Mathematical Finance, Essays in Honour of Jia-an Yan, Eds. T. Zhang and X. Y. Zhou. World Scientific, To appear 2012.

[40] H. Föllmer and I. Penner. Monetary valuation of cash flows under Knightian uncertainty. Int. J. Theor. Appl. Finance, 14(1):1-15, 2011.

[41] H. Föllmer and A. Schied. Convex measures of risk and trading constraints. Finance Stoch., 6(4):429$447,2002$.

[42] H. Föllmer and A. Schied. Stochastic finance-An introduction in discrete time. Graduate Textbook Series. Walter de Gruyter \& Co., Berlin, 3rd edition, 2011.

[43] H. Föllmer, A. Schied, and S. Weber. Robust preferences and robust portfolio choice. In Bensoussan, A., Zhang, (eds.) Handbook of Numerical Analysis, Mathematical Modeling and Numerical Methods in Finance, pages 29-89. 2009.

[44] M. Frittelli and E. Rosazza Gianin. Putting order in risk measures. J. Banking \& Finance, 26:14731486, 2002.

[45] M. Frittelli and E. Rosazza Gianin. Law invariant convex risk measures. Adv. Math. Econ., 7:33-46, 2005.

[46] H. U Gerber. On additive premium calculation principles. Astin Bull., 7(3):215-222, 1974.

[47] H. U. Gerber and M. J. Goovaerts. On the representation of additive principles of premium calculation. Scand. Actuar. J., 4:221-227, 1981.

[48] H. U. Gerber and E. S. W. Shiu. Option pricing by Esscher transforms. Transactions of the society of Actuaries, 46:99-140, 1994.

[49] H. U. Gerber and E. S. W. Shiu. Actuarial bridges to dynamic hedging and option pricing. Insurance Math. Econom., 18(3):183-218, 1996.

[50] I. Gilboa and D. Schmeidler. Maxmin expected utility with nonunique prior. J. Math. Econom., 18(2):141-153, 1989.

[51] M. J. Goovaerts, R. Kaas, J. Dhaene, and Q. Tang. Some new classes of consistent risk measures. Insurance Math. Econom., 34(3):505-516, 2004.

[52] M. J. Goovaerts, R. Kaas, R. J. A. Laeven, and Q. Tang. A comonotonic image of independence for additive risk measures. Insurance Math. Econom., 35(3):581-594, 2004.

[53] M. J. Goovaerts and R. J. A. Laeven. Actuarial risk measures for financial derivative pricing. Insurance Math. Econom., 42(2):540-547, 2008.

[54] J. Haezendonck and M. J. Goovaerts. A new premium calculation principle based on Orlicz norms. Insurance Math. Econom., 1(1):41-53, 1982.

[55] K. F. W. Hattendorff. Über die Berechnung der Reserven und des Risico bei der Lebensversicherung. Masius' Rundschau der Versicherungen, 18:169-183, 1868. 
[56] D. Heath. Back to the future. Plenary Lecture at the First World Congress of the Bachelier Society, Paris, 2000.

[57] F. Hu. On Cramér's theorem for capacities. C. R. Math. Acad. Sci. Paris, 348(17-18):1009-1013, 2010 .

[58] P. J. Huber. Robust statistics. John Wiley \& Sons Inc., New York, 1981. Wiley Series in Probability and Mathematical Statistics.

[59] P. J. Huber and V. Strassen. Minimax tests and the Neyman-Pearson lemma for capacities. Ann. Statist., 1:251-263, 1973.

[60] E. Jouini, M. Meddeb, and N. Touzi. Vector-valued coherent risk measures. Finance Stoch., 8(4):531552,2004

[61] E. Jouini, W. Schachermayer, and N. Touzi. Law invariant risk measures have the Fatou property. Adv. Math. Econ., 9:49-71, 2006.

[62] R. Kaas, M. Goovaerts, J. Dhaene, and M. Denuit. Modern actuarial risk theory. Using R. 2nd ed., 2nd printing. Springer, Berlin, 2009.

[63] M. Kaina and L. Rüschendorf. On convex risk measures on $L^{p}$-spaces. Math. Methods Oper. Res., 69(3):475-495, 2009.

[64] D. M. Kreps. Notes on the theory of choice. Westview Press, Boulder, 1988.

[65] M. Kunze. Verteilungsinvariante konvexe Risikomaße. Diploma thesis, Humboldt-Universität zu Berlin, 2003.

[66] S. Kusuoka. On law invariant coherent risk measures. Adv. Math. Econ., 3:83-95, 2001.

[67] C. C. A. Labuschagne and T. M. Offwood. A note on the connection between the Esscher-Girsanov transform and the Wang transform. Insurance Math. Econom., 47(3):385-390, 2010.

[68] F. Maccheroni, M. Marinacci, and A. Rustichini. Ambiguity aversion, robustness, and the variational representation of preferences. Econometrica, 74(6):1447-1498, 2006.

[69] J. Neveu. Discrete-parameter martingales. North-Holland Publishing Co., Amsterdam, revised edition, 1975. Translated from the French by T. P. Speed, North-Holland Mathematical Library, Vol. 10.

[70] C. Pandit and S. Meyn. Worst-case large-deviation asymptotics with application to queueing and information theory. Stochastic Process. Appl., 116(5):724-756, 2006.

[71] H. H. Panjer, S. S. Wang, and V. R. Young. Axiomatic characterization of insurance prices. Insurance Math. Econom., 21(2):173-183, 1997.

[72] G. Pflug, A. Pichler, and D. Wozabal. The $1 / n$ investment strategy is optimal under high model ambiguity. Journal of Banking \& Finance, 36:410-417, 2012.

[73] G. Pflug and W. Römisch. Modeling, measuring and managing risk. World Scientific Publishing Co. Pte. Ltd., Hackensack, NJ, 2007.

[74] R. T. Rockafellar, S. Uryasev, and M. Zabarankin. Generalized deviations in risk analysis. Finance Stoch., 10(1):51-74, 2006.

[75] L. Rüschendorf. Law invariant convex risk measures for portfolio vectors. Statist. Decisions, 24(1):97108, 2006.

[76] J.S. Sadowsky. Robust large deviations performance analysis for large sample detectors. IEEE Trans. Inform. Theory, 35(4):917-920, 1989.

[77] L. J. Savage. The foundations of statistics. John Wiley \& Sons Inc., New York, 1954.

[78] Y. Song and J.-A. Yan. Risk measures with comonotonic subadditivity or convexity and respecting stochastic orders. Insurance Math. Econom., 45(3):459-465, 2009.

[79] F. Terkelsen. Some minimax theorems. Math. Scand., 31:405-413, 1972.

[80] J. von Neumann and O. Morgenstern. Theory of Games and Economic Behavior. Princeton University Press, Princeton, New Jersey, 1944.

[81] S.S. Wang. Insurance pricing and increased limits ratemaking by proportional hazards transforms. Insurance Math. Econom., 17(1):43-54, 1995. 
[82] S. S. Wang. A class of distortion operators for pricing financial and insurance risks. The Journal of Risk and Insurance, 67(1):15-36, 2000.

[83] S. Weber. Distribution-invariant risk measures, information, and dynamic consistency. Math. Finance, 16(2):419-441, 2006.

[84] M. E. Yaari. The dual theory of choice under risk. Econometrica, 55(1):95-115, 1987.

[85] V. R. Young. Premium Principles. John Wiley \& Sons, New York, Encyclopedia of Actuarial Science edition, 2006. 